\title{
Summary of the 5th Edition of the Renal Association Clinical Practice Guidelines (2009-2012)
}

\author{
Robert Mactier ${ }^{\mathrm{a}}$, Simon Davies ${ }^{\mathrm{b}}$, Chris Dudleyc, Paul Harden ${ }^{\mathrm{d}}$, Colin Jones ${ }^{\mathrm{e}}$, \\ Suren Kanagasundaram ${ }^{\mathrm{f}}$, Andrew Lewington ${ }^{\mathrm{g}}$, Donald Richardson ${ }^{\mathrm{e}}$, Maarten Taal ${ }^{\mathrm{h}}$, \\ Peter Andrews $s^{i}$ Richard Baker ${ }^{9}$, Cormac Breen', Neill Duncan ${ }^{k}$, Ken Farrington', \\ Richard Fluck ${ }^{\mathrm{h}}$, Colin Geddes ${ }^{\mathrm{m}}$, David Goldsmith ${ }^{\mathrm{j}}$, Nic Hoenich ${ }^{\mathrm{n}}$, Stephen Holt ${ }^{\mathrm{O}}$, \\ Alan Jardine ${ }^{p}$, Sarah Jenkins ${ }^{q}$, Mick Kumwendar, Elizabeth Lindleys, Mark MacGregor ${ }^{\mathrm{t}}$, \\ Ashraf Mikhail $^{\mathrm{u}}$, Edward Sharples ${ }^{\mathrm{d}}$, Badi Shresthav, Rajesh Shrivastava ${ }^{\mathrm{w}}$, Simon Steddon ${ }^{\mathrm{j}}$, \\ Graham Warwick $^{\mathrm{x}}$, Martin Wilkie ${ }^{\mathrm{q}}$, Graham Woodrow ${ }^{\mathrm{g}}$, Mark Wright ${ }^{\mathrm{g}}$ \\ ${ }^{a}$ Chair of the Renal Association Clinical Practice Guidelines Committee and consultant nephrologist, NHS Greater \\ Glasgow \& Clyde \\ ${ }^{\mathrm{b}}$ Professor of nephrology, University Hospital of North Staffordshire, Stoke on Trent \\ ${ }^{c}$ Consultant nephrologist, Richard Bright Renal Unit, Bristol \\ ${ }^{\mathrm{d} C}$ Consultant nephrologist, Oxford Kidney Centre, Oxford \\ ${ }^{e}$ Consultant nephrologist, York Hospitals NHS Foundation Trust, York \\ ${ }^{f}$ Consultant nephrologist, Renal Unit, Freeman Hospital, Newcastle \\ ${ }^{9}$ Consultant nephrologist, Leeds Teaching Hospitals, Leeds \\ ${ }^{\mathrm{h}}$ Consultant nephrologist, Derby Hospitals NHS Foundation Trust, Derby \\ 'Consultant nephrologist, SW Thames Renal \& Transplantation Unit, Carshalton, Surrey \\ 'Consultant nephrologist, Guy's and St Thomas' NHS Foundation Trust, London \\ ${ }^{k}$ Consultant nephrologist, Imperial College Healthcare NHS Trust, London \\ 'Professor of nephrology, Lister Hospital, East and North Hertfordshire NHS Foundation Trust \\ ${ }^{m}$ Consultant nephrologist, NHS Greater Glasgow \& Clyde, \\ ${ }^{n}$ Clinical scientist, Renal Unit, Freeman Hospital and Honorary Lecturer, Newcastle University \\ ${ }^{\circ}$ Consultant nephrologist, Sussex Kidney Unit, Brighton \\ PProfessor of nephrology, University of Glasgow \\ ${ }^{\mathrm{q} C}$ Consultant nephrologist, Sheffield Kidney Unit, Sheffield \\ rConsultant nephrologist, Betsi Cadwaladr University Health Board, Glan Glwyd, Rhyl, Wales \\ ${ }^{\mathrm{s}} \mathrm{Clinical}$ scientist, Leeds Teaching Hospitals, Leeds \\ ${ }^{t}$ Consultant nephrologist, Crosshouse Hospital, Kilmarnock \\ ${ }^{u}$ Consultant nephrologist, Morriston Hospital, Swansea \\ ${ }^{\vee}$ Consultant surgeon, Sheffield Kidney Unit, Sheffield \\ ${ }^{w}$ Associate specialist in nephrology, Morriston Hospital, Swansea \\ ${ }^{\times}$Consultant nephrologist, Renal and Urology Directorate, University Hospital Leicester
}

\section{Key Words}

nephrology guidelines - GRADE $\cdot$ Renal Association
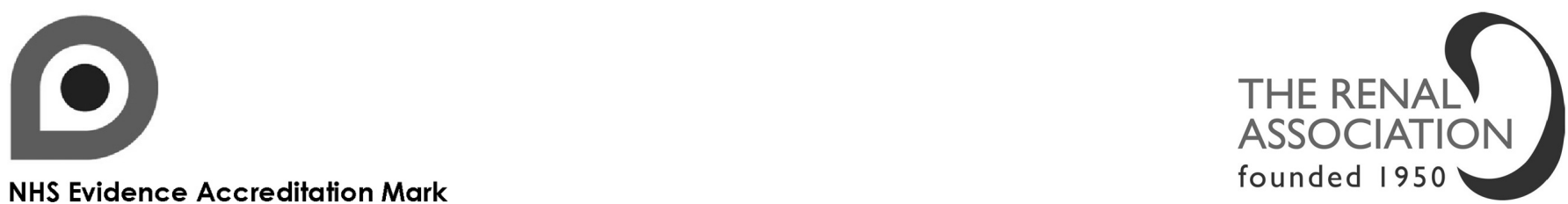

NHS Evidence Accreditation Mark

\section{KARGER}

Fax +4161306 1234

E-Mail karger@karger.ch

www.karger.com
(C) $2011 \mathrm{~S}$. Karger AG, Basel

1160-2110/11/1185-00027\$38.00/0

Accessible online at: www.karger.com/nec 
Summary of Clinical Practice Guideline for Detection, Monitoring and Care of Patients with CKD

\section{Chronic Kidney Disease (CKD) (Guidelines CKD} 1.1-1.12)

\section{Guideline 1.1 - CKD: Detection and Monitoring of} CKD

We recommend that amongst patients with CKD (excluding those on long term dialysis), each measurement of serum creatinine concentration in a database or clinic letter should be accompanied by an estimate of GFR (1B).

\section{Guideline 1.2 - CKD: Detection and Monitoring of CKD}

We recommend that the stage of CKD, as defined by the US K/DOQI classification with the modifications recommended by NICE and/or SIGN, should be noted in the patient records at each clinic visit and communicated in any letters generated (1C).

\section{Guideline 1.3 - CKD: Detection and Monitoring of CKD}

We recommend that in patients being investigated or treated for CKD, proteinuria should be assessed by measurement either of the protein to creatinine or albumin to creatinine ratio, ideally on an early-morning urine specimen $(1 \mathrm{~A})$.

Guideline 1.4 - CKD: Detection and Monitoring of CKD

We recommend that patients who are at increased risk for developing CKD should be offered screening tests to detect CKD (1B).

\section{Guideline 1.5 - CKD: Detection and Monitoring of CKD}

We recommend that screening for CKD should comprise assessment of the estimated GFR as well as urinalysis. (1A)

\section{Guideline 1.6 - CKD: Detection and Monitoring of CKD}

We recommend that detection of an initial abnormal estimated GFR result should prompt clinical assessment and a repeat test within 2 weeks to assess the rate of change in GFR. If the GFR is stable, a further test should be performed after 90 days to confirm the diagnosis of CKD. If the diagnosis of CKD is confirmed at least 3 assessments of estimated GFR should be made over not less than 90 days to evaluate the rate of change in GFR. (1D)

\section{Guideline 1.7 - CKD: Detection and Monitoring of CKD}

We recommend that detection of an initial level of proteinuria equivalent to $<0.5 \mathrm{~g} /$ day of total protein (including levels compatible with microalbuminuria) should be confirmed with a repeat test performed on an early morning urine specimen. For the diagnosis of microalbuminuria 2 abnormal results from 3 specimens are required. (1B)

\section{Guideline 1.8 - CKD: Detection and Monitoring of CKD}

We recommend that patients in whom initial urinalysis reveals non-visible/microscopic haematuria should have a urine culture performed to exclude a urinary tract infection. If a UTI is excluded two further tests should be performed to confirm the presence of persistent non-visible/microscopic haematuria. Patients $>40$ years of age with persistent non-visible/microscopic haematuria in the absence of significant proteinuria or a reduced GFR should be referred to a Urology Department for further investigation. (1D)

\section{Guideline 1.9 - CKD: Detection and Monitoring of CKD}

We recommend that among patients with CKD, the estimated GFR should be monitored regularly. (1B)

\section{Guideline 1.10 - CKD: Detection and Monitoring of CKD}

We recommend that patients with CKD should have the magnitude of their proteinuria assessed at least annually. (1C)

\section{Guideline 1.11 - CKD: Detection and Monitoring of CKD}

We recommend that Nephrology Units should negotiate service agreements for the detection and monitoring of CKD, including criteria for referral to a Nephrologist. (1D)

\section{Guideline 1.12-CKD: Detection and Monitoring of CKD}

We recommend that a Nephrology Unit should establish an easily accessible non-visit-based Specialist advice service for Primary Care Physicians. (1D) 
2. Chronic Kidney Disease (CKD) (Guidelines CKD $2.1-2.13$ )

\section{Guideline 2.1 - CKD: Treatment of patients with CKD}

We recommend that in patients with $\mathrm{CKD}$, systolic blood pressure should be lowered to $<140 \mathrm{mmHg}$ (target range $120-139 \mathrm{mmHg}$ ) and the diastolic blood pressure to $<90 \mathrm{mmHg}$ for the majority. For those with diabetes mellitus or proteinuria of $1 \mathrm{~g} / 24$ hours or greater, the systolic blood pressure should be lowered to $<130 \mathrm{mmHg}$ (target range $120-129 \mathrm{mmHg}$ ) and the diastolic blood pressure to $<80 \mathrm{mmHg}$ unless the risks are considered to outweigh the potential benefits. Antihypertensive therapy should be individualised and lowering the systolic blood pressure to $<120 \mathrm{mmHg}$ should be avoided. (1B)

\section{Guideline 2.2 - CKD: Treatment of patients with CKD}

We recommend that Angiotensin-Converting Enzyme Inhibitor (ACEI) or Angiotensin Receptor Blocker (ARB) treatment should form part of the antihypertensive therapy of patients with CKD and urinary protein excretion of $>0.5 \mathrm{~g} /$ day unless there is a specific contraindication. (1A)

\section{Guideline 2.3 - CKD: Treatment of patients with CKD}

We suggest that patients with CKD and proteinuria $>0.5 \mathrm{~g} /$ day should have their ACEI or ARB and other antihypertensive treatment escalated to achieve the lowest possible level of proteinuria. (2C)

\section{Guideline 2.4 - CKD: Treatment of patients with CKD}

We recommend that patients with diabetes mellitus and microalbuminuria should be treated with an ACEI or ARB, titrated to maximum licensed antihypertensive dose if tolerated, regardless of the initial blood pressure, unless these drugs are specifically contraindicated. Hypotension should be avoided. (1A)

\section{Guideline 2.5 - CKD: Treatment of patients with CKD}

We recommend that patients with diabetes mellitus and CKD should achieve good glycaemic control. (1B)
Guideline 2.6-CKD: Treatment of patients with CKD

We recommend that patients with CKD should have an annual formal assessment of their cardiovascular risk factors including lipid profile, BMI, exercise, alcohol and smoking habits as well as a review of interventions to reduce cardiovascular risk. (1D)

\section{Guideline 2.7 - CKD: Treatment of patients with CKD}

We recommend that patients with CKD and dyslipidaemia should be treated in accordance with current guidance for the general population. (1C)

\section{Guideline 2.8-CKD: Treatment of patients with CKD}

We suggest that smoking status and action taken should be documented in the patient record at each nephrology clinic visit. (1D)

\section{Guideline 2.9-CKD: Treatment of patients with CKD}

We suggest that patients with CKD and a BMI of $>30 \mathrm{~kg} / \mathrm{m}^{2}$ should receive dietary advice to assist them in losing weight. (2D)

\section{Guideline 2.10 - CKD: Treatment of patients with CKD}

We recommend that patients with CKD should receive dietary advice to restrict their sodium intake to $<2.4 \mathrm{~g} /$ day (100 mmol/day or $<6 \mathrm{~g} /$ day of salt). (1B)

\section{Guideline 2.11-CKD: Treatment of patients with CKD}

We recommend that patients with CKD who develop hyperkalaemia or hyperphosphataemia should receive dietary advice to assist dietary restriction of potassium and phosphate. (1C)

\section{Guideline 2.12 - CKD: Treatment of patients with CKD}

We suggest that patients with CKD should receive advice to perform regular moderate exercise. (2D)

\section{Guideline 2.13 - CKD: Treatment of patients with CKD}

We recommend that patients with CKD should be referred for assessment by a Nephrology Department according to the NICE Guidelines (1B). 


\section{Summary of Clinical Practice Guidelines on Anaemia of CKD}

\section{Anaemia of CKD (Guidelines 1.1-1.6)}

Guideline 1.1 - Evaluation of anaemia - Screening for anaemia

We recommend that haemoglobin $(\mathrm{Hb})$ levels should be tested at least annually in CKD patients. (1B)

\section{Guideline 1.2 - Evaluation of anaemia -}

\section{Haemoglobin level}

We recommend that all patients with chronic anaemia associated with chronic kidney disease (CKD) should be investigated for possible treatment, irrespective of the stage of kidney disease or requirement for renal replacement therapy. (1A)

Anaemia should be evaluated in CKD patients with $\mathrm{Hb}<11 \mathrm{~g} / \mathrm{dL}$ or symptoms attributable to anaemia.

\section{Guideline 1.3 - Evaluation of anaemia - Renal function}

We suggest that CKD should be considered as a possible cause of anaemia when the glomerular filtration rate (GFR) is $<60 \mathrm{ml} / \mathrm{min} / 1.73 \mathrm{~m}^{2}$. It is more likely to be the cause if the GFR is $<30 \mathrm{mls} / \mathrm{min} / 1.73 \mathrm{~m}^{2}(<45$ in diabetics) and no other cause, i.e. blood loss, folic acid or vitamin B12 deficiency, is identified. (2B)

\section{Guideline 1.4 - Evaluation of anaemia -}

\section{Erythropoietin measurement}

We recommend that measurement of erythropoietin levels should not routinely be considered for the diagnosis or management of anaemia for patients with CKD. (1A)

\section{Guideline 1.5 - Evaluation of anaemia - Baseline investigations}

We recommend that initial clinical and laboratory evaluation of anaemia should be performed prior to initiation of treatment for anaemia in CKD patients. (1A)

\section{Guideline 1.6 - Evaluation of anaemia - Baseline investigations}

We recommend that laboratory evaluation should include the following tests (1B):

- Full blood count (FBC) including - in addition to the $\mathrm{Hb}$ concentration - red blood cell indices (mean corpuscular haemoglobin $[\mathrm{MCH}]$, mean corpuscular volume $[\mathrm{MCV}]$, mean corpuscular haemoglobin concentration $[\mathrm{MCHC}])$, white blood cell count, differential count and platelet count.

- Absolute reticulocyte count to assess bone marrow responsiveness (if indicated).

- Serum ferritin to assess iron stores.

- Serum transferrin saturation (TSAT) or reticulocyte $\mathrm{Hb}$ content $(\mathrm{CHr})$ to assess adequacy of iron for erythropoiesis.

- Percentage of hypochromic red blood cells (HRC).

- Plasma/serum C-reactive protein (CRP) - to assess inflammation.

Based on the initial assessment and in selected cases, the following tests may be useful to diagnose the cause of anaemia in some patients with CKD:

- Serum B12 and red cell folate concentrations.

- Tests for haemolysis (plasma/serum levels of haptoglobin, lactate dehydrogenase, bilirubin, Coombs' test).

- Plasma/serum and/or urine protein electrophoresis.

- Hb electrophoresis and bone marrow examination.

\section{Anaemia of CKD (Guidelines 2.1-2.10)}

Guideline 2.1 - Treatment of Anaemia Erythropoiesis Stimulating Agents

We recommend that treatment with Erythropoiesis Stimulating Agents (ESAs) should be offered to patients with anaemia of CKD who are likely to benefit in terms of quality of life and physical function and to avoid transfusion in patients considered suitable for transplantation. (1A)

\section{Guideline 2.2 - Treatment of Anaemia - Choice of} ESA

We recommend that the decision on the choice of ESA is based on local availability of ESAs. (1B)

\section{Guideline 2.3 - Treatment of Anaemia with ESA} therapy - Target $\mathrm{Hb}$

We recommend that patients with CKD on ESA therapy should achieve Hb between 10-12 g/dl. (1B)

Guideline 2.4 - Treatment of Anaemia without ESA therapy - Target $\mathrm{Hb}$

We recommend that these $\mathrm{Hb}$ targets apply exclusively to patients receiving ESA and are not intended to apply to the treatment of iron deficiency in patients receiving iron therapy without the use of ESAs. (1B) 
Guideline 2.5 - Treatment of Anaemia - Initial ESA dose

We recommend that the initial ESA dose should be determined by the patient's $\mathrm{Hb}$ level, the target $\mathrm{Hb}$ level, the observed rate of increase in $\mathrm{Hb}$ level and clinical circumstances. (1B)

\section{Guideline 2.6 - Treatment of Anaemia with ESA} therapy - Route of administration

We suggest that the route of ESA administration should be determined by the CKD stage, treatment setting, efficacy, safety, and class of ESA used; subcutaneous (SC) route is the access of choice in non-HD patients, while convenience may favour intravenous (IV) administration in HD patients. (2B)

Guideline 2.7 - Treatment of Anaemia with ESA therapy - Frequency of administration

We suggest that the frequency of administration should be determined by the CKD stage, treatment setting and class of ESA. Less frequent administration using long acting ESAs may be the treatment of choice in non-HD patients. (2B)

Guideline 2.8 - Treatment of Anaemia with ESA Therapy - ESA dose adjustments

We recommend that adjustments to ESA doses should be considered when $\mathrm{Hb}$ is $<10.5$ or $>11.5 \mathrm{~g} / \mathrm{dL}$ in order to balance benefit and safety to patients given the current evidence base. These thresholds for intervention should achieve a population distribution centred on a mean of $11 \mathrm{~g} / \mathrm{dl}$ with a range of $10-12$. (1B)

Guideline 2.9 - Treatment of Anaemia with ESA Therapy - ESA dose adjustments

We suggest that ESA doses should ideally be decreased rather than withheld when a downward adjustment of $\mathrm{Hb}$ level is needed. (2B)

\section{Guideline 2.10 - Treatment of Anaemia with ESA}

Therapy - ESA dose adjustments

We recommend that ESA administration in ESAdependent patients should continue during acute illness, surgical procedures or any other cause of hospitalisation. (1B)

\section{Anaemia of CKD (Guidelines 3.1-3.4)}

Guideline 3.1 - Treatment of Anaemia with Iron therapy - Iron repletion

We recommend that patients should be iron replete to achieve and maintain target $\mathrm{Hb}$ whether receiving ESAs or not. (1A)

Guideline 3.2 - Treatment of Anaemia with Iron therapy - Initiation of ESA and iron status

We recommend that ESA therapy should not be initiated in the presence of absolute iron deficiency (ferritin $<100 \mathrm{ng} / \mathrm{ml}$ ). In patients with functional iron deficiency, iron supplements should be given prior to or when initiating ESA therapy. (1A)

Guideline 3.3 - Treatment of Anaemia with Iron therapy - Route of Administration

We suggest that oral iron will, in general, be sufficient to attain and maintain the $\mathrm{Hb}$ above targets in ESA treated CKD patients not yet requiring dialysis and in those on peritoneal dialysis (PD). (2B)

In contrast most $\mathrm{HD}$ patients will require intravenous iron. $(2 \mathrm{~A})$

\section{Guideline 3.4 - Treatment of Anaemia with Iron}

\section{therapy - Upper limit for iron therapy}

We recommend that serum ferritin should not exceed $800 \mathrm{ng} / \mathrm{ml}$ in patients treated with iron, and to achieve this iron management should be reviewed when the ferritin is $>500 \mathrm{ng} / \mathrm{ml}$. (1B)

\section{Anaemia of CKD (Guidelines 4.1-4.5)}

\section{Guideline 4.1 - Monitoring of Treatment - Hb} during ESA therapy

We recommend that $\mathrm{Hb}$ concentration should be monitored every month in the correction phase and every 1-3 months for stable patients in the maintenance phase. More frequent monitoring will depend on clinical circumstances. (1B)

\section{Guideline 4.2 - Monitoring of Treatment - Iron therapy}

We recommend regular monitoring of iron status (1-3 monthly) during treatment to avoid toxicity (1B): a serum ferritin consistently greater than $800 \mathrm{ng} / \mathrm{ml}$ is suggestive of iron overload. (1B)

Guideline 4.3 - Monitoring during Intravenous Iron Administration

We recommend that resuscitative medication and personnel trained to evaluate and resuscitate anaphylaxis should be present at each administration of iron dextran. (1A) 
Guideline 4.4 - Monitoring of Treatment Resistance to ESA therapy

We recommend that inadequate response ('resistance') to ESA therapy is defined as failure to reach the target $\mathrm{Hb}$ level despite SC epoetin dose $>300 \mathrm{IU} / \mathrm{kg} /$ week (450 IU/ $\mathrm{kg} /$ week IV epoetin), or darbepoetin dose $>1.5 \mathrm{mcg} / \mathrm{kg} /$ week. Hyporesponsive patients who are iron replete should be screened clinically and by investigations for other common causes of anaemia. (1A)

\section{Guideline 4.5 - Monitoring of treatment -} Hypertension during ESA therapy

We recommend that blood pressure should be monitored in all patients receiving ESAs and, if present, hypertension be treated by volume removal and/or hypotensive drugs. (1A)

\section{Anaemia of CKD (Guidelines 5.1-5.2)}

\section{Guideline 5.1 - Blood Transfusion}

We recommend that in patients with anaemia of CKD, especially those in whom renal transplantation is an option, red blood cell transfusion should be avoided if possible. (1A)

\section{Guideline 5.2 - Blood Transfusion}

We recommend if red blood cell transfusion becomes essential (usually in the setting of acute blood loss, acute haemolysis or severe sepsis) transfusion should be based on policies set by local transfusion guidelines rather than $\mathrm{Hb}$ targets for ESA therapy in chronic anaemia of CKD. (1B)

\section{Anaemia of CKD (Guideline 6.1)}

\section{Guideline 6.1 - Post-transplantation Anaemia}

We recommend that the treatment guidelines for anaemia in renal transplant patients should be similar to those for CKD patients not on dialysis. (1B) 


\section{Summary of Clinical Practice Guidelines for Cardiovascular Disease in CKD}

\section{Cardiovascular disease in CKD (CVD) (Guidelines CVD 1.1-1.8)}

\section{Guideline 1.1 - CVD: Cardiovascular risk factors}

We recommend that a history of and risk factors for cardiovascular disease in patients with CKD stage 1-5 and dialysis patients should be recorded in a format that permits audit of the management of such patients (1B).

These should include:

- Angina and myocardial infarction

- Previous coronary angioplasty or coronary artery bypass grafting

- Stroke and transient ischaemic attack

- Previous carotid artery surgery or angioplasty

- Peripheral vascular disease or previous intervention

- Cardiac failure

- Arrhythmias (supraventricular and ventricular)

- Diabetes

- Ethnicity

\section{Guideline 1.2 - CVD: Cardiovascular risk factors}

We recommend that a healthy lifestyle should be encouraged in all CKD patients, including dialysis patients. (1C)

\section{Guideline 1.3 - CVD: Cardiovascular risk factors}

We suggest that smoking habits should be recorded and smoking should be actively discouraged in all patients with a reasonable life expectancy and strongly discouraged in those patients on the transplant waiting list. (2B)

\section{Guideline 1.4 - CVD: Cardiovascular risk factors}

We suggest that exercise should be encouraged and patients, including dialysis patients, should be enrolled on regular exercise programmes, exercising 3 to 5 times weekly either during dialysis or between dialysis sessions. (2C)

\section{Guideline 1.5 - CVD: Cardiovascular risk factors}

We suggest that the target glycated haemoglobin $\left(\mathrm{HbAl}_{\mathrm{c}}\right)$ in all CKD, dialysis and transplant patients with diabetes should be between $6.5 \%$ (48 mmol/ $\left.\mathrm{mmol} / \mathrm{HbA}_{0}\right)$ and $7.5 \%\left(58 \mathrm{mmol} / \mathrm{mmol} / \mathrm{HbA}_{0}\right)$. (2C)
Guideline 1.6 - CVD: Cardiovascular risk factors

We recommend that statins (or 3 hydroxy-3methylglutaryl-coenzyme A reductase inhibitors) should be considered for primary prevention in all CKD stages 14 and transplant patients with a 10-year risk of cardiovascular disease, calculated as $>20 \%$ according to the Joint British Societies' Guidelines - JBS2-(British Hypertension Society British Cardiac Society 2005). (1B)

\section{Guideline 1.7 - CVD: Cardiovascular risk factors}

We recommend that a total cholesterol of $<4 \mathrm{mmol} / \mathrm{l}$ or a $25 \%$ reduction from baseline, or a fasting low density lipoprotein (LDL)-cholesterol of $<2 \mathrm{mmol} / \mathrm{l}$ or a $30 \%$ reduction from baseline, should be achieved, whichever is the greatest reduction in all patients. (1B)

\section{Guideline 1.8 - CVD: Cardiovascular risk factors}

We suggest that statins should not be withdrawn from patients in whom they were previously indicated and should continue to be prescribed when such patients start renal replacement therapy (RRT) or change modality. (2C)

\section{Cardiovascular disease in CKD (CVD) (Guidelines CVD 2.1-2.3)}

\section{Guideline 2.1 - CVD: B vitamin and folate supplementation}

We suggest that folic acid and B vitamin supplements should be offered to all renal patients considered nutritionally at risk from deficiency of folic acid or B vitamin deficiency. B12 levels and, serum and red cell folate should be above the lower limit of the reference range in all CKD patients including patients on dialysis and after transplantation. (2C)

\section{Guideline 2.2 - CVD: Folate deficiency}

We suggest that red cell folate levels should be checked if $\mathrm{MCV}$ remains high despite normal or high serum folate. $(2 \mathrm{C})$

Guideline 2.3 - CVD: Hyperhomocysteinaemia and vitamin supplementation

We suggest that serum folate levels and B12 should be checked 6 monthly in CKD4/5 and 3 monthly in dialysis patients or more frequently if patients remain anaemic or deficient on initial sampling. There is insufficient evidence of the effects of these vitamins on modifying vascular risk by effects on homocysteine in dialysis patients to recommend supraphysiological replacement. (2D) 


\section{Cardiovascular disease in CKD (CVD) (Guidelines CVD 3.1-3.6)}

\section{Guideline 3.1 - CVD: Secondary prevention of cardiovascular risk}

We recommend that CKD stage $1-3$ patients with a history of chronic stable angina, acute coronary syndrome, myocardial infarction, stroke, peripheral vascular disease, or who undergo surgical or angiographic coronary revascularisation, should be prescribed aspirin, an ACE inhibitor, a beta-blocker, and an HMG-CoA reductase inhibitor unless contraindicated as per NICE Guidance. (1B)

\section{Guideline 3.2 - CVD: Secondary prevention of cardiovascular risk}

We suggest that CKD stage $4 / 5$ patients (including those on dialysis and after transplantation) with a history of chronic stable angina, acute coronary syndrome, myocardial infarction, stroke, peripheral vascular disease, or who undergo surgical or angiographic coronary revascularisation, should be prescribed aspirin, an ACE inhibitor, a beta-blocker, and an HMG-CoA reductase inhibitor unless contraindicated as per NICE Guidance. $(2 \mathrm{C})$

\section{Guideline 3.3 - CVD: Secondary prevention of cardiovascular risk}

We suggest that aspirin and clopidogrel may be indicated for up to 12 months post angioplasty and stenting and in non-ST elevation MI but may have an excess of bleeding complications. (2C)

\section{Guideline 3.4 - CVD: Secondary prevention of cardiovascular risk}

We suggest that aspirin is indicated for secondary prevention but not primary prevention of vascular disease in renal failure. $(2 \mathrm{C})$

\section{Guideline 3.5 - CVD: Secondary prevention of cardiovascular risk}

We suggest that the doses of ACE inhibitors and betablockers should be titrated upwards to the maximal effective or tolerated dose. (2C)

\section{Guideline 3.6 - CVD: Secondary prevention of cardiovascular risk}

We suggest that patients on lipid-lowering drug treatment should have total cholesterol reduced by $25 \%$ or to below $4 \mathrm{mmol} / \mathrm{l}$, or LDL-cholesterol to below
$2 \mathrm{mmol} / \mathrm{l}$, or reduced by $30 \%$, whichever reductions are the greatest. (2B)

\section{Cardiovascular disease in CKD (CVD) (Guidelines CVD 4.1-4.3)}

\section{Guideline 4.1 - CVD: Cardiac investigations and coronary revascularisation}

We suggest that CKD and dialysis patients should have unimpeded access to a full range of cardiac investigations including exercise and stress echocardiography, radioisotopic cardiac scans, and coronary angiography. They should also have unimpeded access to cardiology assessment for coronary angioplasty, coronary stenting and cardiac surgery. (2D)

\section{Guideline 4.2 - CVD: Cardiac investigations and coronary revascularisation}

We suggest that there should be no clinically important delay for pre-dialysis and dialysis patients in receiving assessment by cardiology colleagues for their suitability for transplantation. These issues are often best addressed by regular/joint working with other disciplines. (2D)

\section{Guideline 4.3 - CVD: Cardiac investigations and coronary revascularisation}

We suggest that the patient's view of the risk and benefit in deciding whether to undergo complex procedures, including renal transplantation, should always carry significant weight in the eventual decisions reached. (2D)

\section{Cardiovascular disease in CKD (CVD) (Guidelines CVD 5.1-5.7)}

\section{Guideline 5.1 - CVD: Hypertension in non-dialysis patients}

We suggest that $\mathrm{BP}$ in $\mathrm{CKD} 1-4$ should be managed according to NICE guidance: $<140 / 90$ in patients without significant proteinuria and $<130 / 80$ in those with proteinuria or with diabetes. $(2 \mathrm{C})$

\section{Guideline 5.2 - CVD: Hypertension in dialysis patients}

We suggest that pre- and post-dialysis blood pressure (measured after completion of dialysis, including washback) should be recorded and intra-dialytic blood pressure measurements should be made to facilitate good management of the HD session. (2D) 


\section{Guideline 5.3 - CVD: Hypertension in dialysis patients}

We suggest that home or ambulatory blood pressure recordings should be performed if pre- and post-HD or clinic blood pressures are regularly elevated ( $>160 \mathrm{mmHg}$ systolic BP for $>50 \%$ of the recording period) or there is concern over possible hypotension. $(2 \mathrm{C})$

\section{Guideline 5.4 - CVD: Hypertension in dialysis patients}

Blood pressure targets for dialysis patients are difficult to recommend in the absence of RCTs showing survival benefit, and even more difficult to achieve in practice. However we suggest that it would be sensible to avoid sustained BP extremes and, in order to try to provide some guidance we suggest that systolic blood pressure during the inter-dialytic period on $\mathrm{HD}$, and for $\mathrm{PD}$ patients, should not regularly exceed $>160 \mathrm{mmHg}$. (2C)
Guideline 5.5 - CVD: Hypotension/Hypertension in dialysis patients

We suggest that systolic blood pressure should not routinely be treated with pharmacological agents with antihypertensive properties if SBP is regularly $<120 \mathrm{mmHg}$ pre dialysis. Discussion with cardiological colleagues may be prudent if ACEI, ARB or BB are being used for LV systolic or diastolic dysfunction in the context of low BP. (2D)

\section{Guideline 5.6 - CVD: Hypertension in dialysis} patients

We suggest that dialysis patients should be on a restricted salt ( $<6 \mathrm{~g} /$ day $)$ diet. $(2 \mathrm{C})$

\section{Guideline 5.7 - CVD: Hypertension in dialysis patients}

We suggest that hypertension on dialysis should be managed by ultrafiltration in the first instance. (2D) 
Summary of Clinical Practice Guidelines on Mineral and Bone Disorders in CKD

\section{CKD-Mineral and Bone Disorders (Guideline CKD-MBD 1.1)}

Guideline 1.1 CKD-MBD: Monitoring of biochemical parameters

We suggest that serum levels of calcium, phosphate, alkaline phosphatase, PTH and calcidiol $(25(\mathrm{OH}) \mathrm{D})$ should be monitored in patients with CKD stage 3-5, and patients on dialysis, with a frequency based on stage, rate of progression and whether specific therapies have been initiated. In general, it is recommended that therapeutic decisions are based on trends, rather than a single laboratory value, and that they take into account the entire available dataset, rather than isolated variables (not graded).

\section{CKD-Mineral and Bone Disorders (Guidelines} CKD-MBD 2.1-2.2)

Guideline 2.1 CKD-MBD: Serum calcium in patients with CKD stage 3-5 (not on dialysis)

We suggest that serum calcium, adjusted for albumin concentration, in patients with CKD stage 3-5 should be kept within the normal reference range for the laboratory used. (2D)

Guideline 2.2 CKD-MBD: Serum calcium in dialysis patients (stage 5D)

We suggest that serum calcium, adjusted for albumin concentration, should be maintained within the normal reference range for the laboratory used, measured before a 'short-gap' dialysis session in haemodialysis patients. Ideally, adjusted serum calcium should be maintained between 2.2 and $2.5 \mathrm{mmol} / \mathrm{L}$, with avoidance of hypercalcaemic episodes. (2D)

\section{CKD-Mineral and Bone Disorders (Guidelines CKD-MBD 3.1-3.2)}

Guideline 3.1 CKD-MBD: Serum phosphate in patients with CKD 3-5 (not on dialysis)

We suggest that serum phosphate in patients with
CKD stage $3 \mathrm{~b}-5$ should be maintained between 0.9 and $1.5 \mathrm{mmol} / \mathrm{L} .(2 \mathrm{C})$

Guideline 3.2 CKD-MBD: Serum phosphate in dialysis patients (stage $5 \mathrm{D}$ )

We suggest that serum phosphate in dialysis patients, measured before a 'short-gap' dialysis session in haemodialysis patients, should be maintained between 1.1 and $1.7 \mathrm{mmol} / \mathrm{L}$. (2C)

\section{CKD-Mineral and Bone Disorders (Guidelines CKD-MBD 4.1-4.2)}

\section{Guideline 4.1 CKD-MBD: Serum PTH in patients} with CKD $3 b-5$ (not on dialysis)

We suggest that treatment is considered in patients with CKD stages $3 \mathrm{~b}-5$ not on dialysis therapy in whom serum PTH levels are progressively increasing and remain persistently higher than the upper reference limit for the assay, despite correction of modifiable factors. (2C)

Guideline 4.2 CKD-MBD: Serum PTH in patients on dialysis (stage 5D)

\section{Guideline 4.2.1 CKD-MBD: Target range of serum $P T H$ in patients on dialysis}

We suggest that the target range for parathyroid hormone measured using an intact PTH assay should be between 2 and 9 times the upper limit of normal for the assay used. (2C)

Guideline 4.2.2 CKD-MBD: Trends in serum PTH in patients on dialysis

We suggest that marked changes in PTH levels in either direction within this range should prompt an initiation or change in therapy to avoid progression to levels outside this range. (2C) 


\section{Summary of Clinical Practice Guidelines for Nutrition} in CKD

\section{Screening for undernutrition in CKD (Guidelines 1.1-1.2)}

\section{Guideline 1.1.1 - Screening methods for undernutrition in CKD}

We recommend that all patients with stage 4-5 CKD should have the following parameters measured as a minimum in order to identify undernutrition (1C):

- Actual Body Weight (ABW) $(<85 \%$ of Ideal Body Weight (IBW))

- Reduction in oedema free body weight (of 5\% or more in 3 months or $10 \%$ or more in 6 months)

- BMI $\left(<20 \mathrm{~kg} / \mathrm{m}^{2}\right)$

- Subjective Global Assessment (SGA) (B/C on 3 point scale or $1-5$ on 7 point scale)

The above simple audit measures have been linked to increased mortality and other adverse outcomes.

Guideline 1.1.2 - Additional methods for assessment of undernutrition in CKD

We suggest that other measures including bioimpedance analysis, anthropometry, handgrip strength and assessment of nutrient intake can help to further assess nutritional state in those who are at risk of developing or have developed undernutrition. (2B)

Low serum albumin is a strong predictor of adverse outcomes, but it is largely unrelated to nutritional status.

\section{Guideline 1.2 - Frequency of screening for undernutrition in CKD}

We recommend that screening should be performed. (1D):

- Weekly for inpatients

- 2-3 monthly for outpatients with eGFR $<20$ but not on dialysis

- Within one month of commencement of dialysis then $6-8$ weeks later

- 4-6 monthly for stable haemodialysis patients

- 4-6 monthly for stable peritoneal dialysis patients

Screening may need to occur more frequently if risk of undernutrition is increased (for example by intercurrent illness)

\section{Prevention of undernutrition in CKD (Guidelines} 2.1-2.6)

\section{Guideline 2.1 - Dose of small solute removal to} prevent undernutrition

We recommend that dialysis dose meets recommended solute clearance index guidelines (e.g. URR, $\mathrm{Kt} / \mathrm{V})$. (1C)

Guideline 2.2 - Correction of metabolic acidosis and nutrition

We recommend that venous bicarbonate concentrations should be maintained above $22 \mathrm{mmol} / \mathrm{l}$. (1C)

\section{Guideline 2.3 - Minimum daily dietary protein intake}

We suggest a prescribed protein intake of:

- $0.75 \mathrm{~g} / \mathrm{kg}$ IBW/day for patients with stage 4-5 CKD not on dialysis

- $1.2 \mathrm{~g} / \mathrm{kg}$ IBW/day for patients treated with dialysis. (2B)

Recommended nutrient intakes are designed to ensure that $97.5 \%$ of a population take in enough protein and energy to maintain their body composition. There is variation in actual nutrient requirement between individuals. This means that some patients will be well maintained with lower nutrient intakes. Regular screening will help to identify when the dietary prescription needs to be amended.

\section{Guideline 2.4 - Recommended daily energy intake}

We suggest a prescribed energy intake of

- 30-35 kcal/kg IBW/day for all patients depending upon age and physical activity. (2B)

Recommended nutrient intakes are designed to ensure that $97.5 \%$ of a population take in enough protein and energy to maintain their body composition. There is variation in actual nutrient requirement between individuals. This means that some patients will be well maintained with lower nutrient intakes. Regular screening will help to identify when the dietary prescription needs to be amended.

\section{Guideline 2.5 - Vitamin supplementation in dialysis patients}

We recommend that dialysis patients should be prescribed supplements of water soluble vitamins. (1C) 
Guideline 2.6 - Exercise programs in dialysis patients

We recommend that haemodialysis patients should be given the opportunity to participate in regular exercise programmes. (1C)

Progressive resistance training and aerobic exercise have both been shown to bring about improvement in physical function and some components of Quality of Life scores.

\section{Treatment of established undernutrition in CKD (Guidelines 3.1-3.6)}

\section{Guideline 3.1 - General treatment of established undernutrition}

We recommend assessment by a physician to determine and treat possible underlying causes and by a specialist dietician to individualise dietary advice. (1D)

\section{Guideline 3.2 - Oral nutritional supplements in established undernutrition}

We recommend the use of oral nutritional supplements if oral intake is below the levels indicated above and food intake cannot be improved following dietetic intervention. (1C)

Guideline 3.3 - Enteral nutritional supplements in established undernutrition

We recommend the use of enteral feeding via NG tube/PEG if nutrient intake is suboptimal despite oral supplements. (1C)

Guideline 3.4 - Parenteral nutritional supplements in established undernutrition

We suggest intradialytic parenteral nutrition (IDPN) or intraperitoneal amino acids may be considered for selected cases if tube feeding is declined or clinically inappropriate. (2D)

Guideline 3.5 - Anabolic agents in established undernutrition

We recommend that anabolic agents such as androgens, growth hormone or IGF-1. are not indicated in the treatment of undernutrition in adults. (1D)

Androgens and growth hormone have demonstrated improvement in serum albumin levels and lean body mass but not mortality and these medications have significant side effects.

\section{Guideline 3.6 - Supplementation of micronutrients} in established undernutrition

We suggest that current evidence does not support the routine use of micronutrient supplements other than for identified clinical need. (2C)

While deficiencies of fat soluble vitamins, trace elements and carnitine are prevalent in patients with chronic kidney disease current evidence does not support either preventative or therapeutic supplementation. However emerging evidence may suggest that supplementation of oral vitamin $\mathrm{D}$ (either cholecalciferol or ergocalciferol) is beneficial. (2C)

\section{Overnutrition in CKD (Guidelines 4.1-4.2)}

\section{Guideline 4.1 - Monitoring overnutrition}

We recommend that obesity can be assessed by BMI. (1C)

\section{Guideline 4.2 - Monitoring overnutrition}

We suggest that waist and hip circumferences should not be collected routinely in CKD patients at this time. $(2 \mathrm{C})$ 
Summary of Clinical Practice Guidelines on Prevention of Blood Borne Virus Infection in CKD

\section{Blood Borne Virus Infection (BBV Infection) (Guidelines BBV 1.1-1.2)}

Guideline 1.1 - BBV Infection: Prevention of spread to patients and staff in the renal unit (Universal precautions)

We recommend that infection-control procedures should include hygienic precautions that effectively prevent the transfer of blood or fluids contaminated with blood between patients either directly or via contaminated equipment or surfaces (often referred to as 'universal precautions'). (1A) (KDIGO Hepatitis C Guideline 3.2)

Guideline 1.2 - BBV Infection: Prevention of spread to patients and staff in the renal unit

We recommend that medicine vials should be discarded after single use or, if used for more than one patient, divided into multiple doses and distributed from a central area. (1B)

\section{Blood Borne Virus Infection (BBV Infection) \\ (Guidelines BBV 2.1-2.5)}

\section{Guideline 2.1 - BBV Infection: Dialysis equipment}

We recommend that separate machines should be used for patients known to be infected with HBV (or at high risk of new HBV infection - see section 3.7 and 3.8 below). A machine that has been used for HBV patients can be used again for non-infected patients only after it has been thoroughly decontaminated. (1A)

\section{Guideline 2.2 - BBV Infection: Dialysis equipment}

We recommend that dedicated machines are not required for patients with HCV or HIV provided that disinfection processes are properly carried out between patients according to a local protocol that incorporates the manufacturer's instructions. (1B) (KDIGO Hepatitis C guideline 3.1)

Guideline 2.3 - BBV Infection: Dialysis equipment

We recommend that dialysers manufactured for multiple use can be re-used provided there is implementation of, and adherence to, strict infection control procedures to avoid dialysers or blood port caps being switched between patients. (1C) (KDIGO Hepatitis C guideline 3.1)
Guideline 2.4-BBV Infection: Dialysis equipment

We suggest that external transducer protectors on the blood circuit pressure monitoring lines should be inspected by healthcare personnel during and after each dialysis session. If there is evidence of breach by blood or saline then the machine should be taken out of service and machine components that may have come in contact with blood should be replaced or decontaminated by qualified personnel according to a protocol that incorporates the manufacturers' instructions. (2C)

\section{Guideline 2.5 - BBV Infection: Dialysis equipment}

We recommend that the dialysis machine should be cleaned between patients according to a local protocol that incorporates the manufacturer's instructions. (1C)

\section{Blood Borne Virus Infection (BBV Infection) (Guidelines BBV 3.1-3.12)}

Guideline 3.1 - BBV Infection: BBV surveillance in dialysis patients

We recommend that all patients starting haemodialysis (including patients with acute kidney injury) or returning to haemodialysis after another modality of renal replacement therapy should be known to be plasma HBV surface antigen ( $\mathrm{HbsAg}$ ) negative before having dialysis on the main dialysis unit. (1A)

Guideline 3.2 - BBV Infection: BBV surveillance in dialysis patients

We recommend that patients who require haemodialysis before the result of the HBsAg test is known should be dialysed in an area that is segregated from the main dialysis unit and the machine should not be used for another patient until the result is known to be negative or the machine has been decontaminated (see 2.1). (1A)

Guideline 3.3 - BBV Infection: BBV surveillance in dialysis patients

We recommend that patients on regular hospital haemodialysis who have responded to hepatitis B immunisation (annual anti HBs antibody titre $>10 \mathrm{mIU} / \mathrm{ml}$; see section 5 below), only need to be tested for HBsAg once a year. Non-responders should be tested at least every 3 months. (1C)

Guideline 3.4 - BBV Infection: BBV surveillance in dialysis patients

We recommend that patients on regular hospital haemodialysis should be tested for HCV antibody at

Nephron Clin Pract 2011;118(suppl 1):c27-c70 
least every 6 months. (1C) (KDIGO Hepatitis C guideline 1.2.2)

Guideline 3.5 - BBV Infection: BBV surveillance in dialysis patients

We recommend that antibody surveillance testing for HIV is not necessary for patients on regular hospital haemodialysis unless the patient is at high risk (see Table 2 in full guideline). (1C)

Guideline 3.6 - BBV Infection: BBV surveillance in dialysis patients

We suggest that patients who do not consent to BBV surveillance as described above should have dialysis in a segregated area unless they are known to be HBV immune. If patients who are known to be $\mathrm{HBV}$ immune do not consent to BBV surveillance then they should be managed in the same way as patients with $\mathrm{HCV}$ infection (see section 4). (2C)

Guideline 3.7 - BBV Infection: BBV surveillance in dialysis patients

We recommend that patients returning from dialysing outside the UK should have a risk assessment for potential exposure to BBV abroad. Where exposure is considered likely, enhanced surveillance testing for BBV should be instituted and patients should have dialysis in a segregated area until the HbsAg is known to be negative (see 3.8 below) unless they are known to be HBV immune (antiHBs $>100 \mathrm{mIU} / \mathrm{mL}$ within the last 12 months). (1B)

\section{Guideline 3.8 - BBV Infection: BBV surveillance in dialysis patients}

We recommend that enhanced surveillance in patients deemed to be at high risk after returning from abroad should consist of HCV RNA (or HCV core antibody) every 2 weeks for 3 months and, if not known to be $\mathrm{HBV}$ immune (anti-HBs $>100 \mathrm{mIU} / \mathrm{mL}$ within the last 12 months), HbsAg (or plasma HBV DNA) every 2 weeks for 3 months. Nucleic acid testing (NAT) for $\mathrm{HCV}$ and $\mathrm{HBV}$ in the first 1-2 months virtually excludes acute infection. If HIV is a possibility screening with 4th generation (antigen/antibody combination) assays should be used. (1B)

\section{Guideline 3.9 - BBV Infection: BBV surveillance in dialysis patients}

We recommend that patients at high risk for new BBV infection (see Table 2 in full guideline) should also have enhanced surveillance as described in 3.8. (1B)
Guideline 3.10 - BBV Infection: BBV surveillance in dialysis patients

We recommend that testing for HBV DNA and HCV RNA should be performed in haemodialysis patients with unexplained abnormal serum aminotransferase concentrations. (1B) (KDIGO Hepatitis C guideline 1.2.3)

\section{Guideline 3.11 - BBV Infection: BBV surveillance in} dialysis patients

We recommend that, if a new BBV infection in a haemodialysis unit is identified, testing for viral RNA or DNA should be performed in all patients who may have been exposed (see section 7). (1B) (KDIGO Hepatitis $\mathrm{C}$ guideline 1.2.4)

Guideline 3.12 - BBV Infection: BBV surveillance in dialysis patients

We recommend that testing for HCV antibody should be by third generation ELISA. (1B)

\section{Blood Borne Virus Infection (BBV Infection) (Guidelines BBV 4.1-4.3)}

\section{Guideline 4.1 - BBV Infection: Segregation of patients infected with $B B V$ or at high risk for new $B B V$ infection}

We recommend that patients infected with HBV should be dialysed in an area that is segregated from the main dialysis unit. (1A)

\begin{abstract}
${ }^{a}$ The DoH report 2002 defined segregation between infected and clean areas in a renal unit as being 'functionally complete with no possibility of traffic between the two' and suggested there be a physical barrier such as walls or screens between these infected and clean areas.
\end{abstract}

Guideline 4.2 - BBV Infection: Segregation of patients infected with $B B V$ or at high risk for new $B B V$ infection

We recommend that healthcare workers performing dialysis on patients infected with HBV infection, whenever possible, should not dialyse patients without HBV infection at the same time. (1C)

\section{Guideline 4.3 - BBV Infection: Segregation of} patients infected with $B B V$ or at high risk for new $B B V$ infection

We recommend that patients with HCV or HIV do not need to be dialysed in a segregated area but more experienced staff should be allocated to dialyse these patients. (1C) If nosocomial transmission continues to 
occur, despite reinforcement and audit of the precautions listed in guidelines 1.1 and 1.2, a local segregation policy may be deemed necessary. HCV-infected patients should then be treated by dedicated staff in a separate room, area, or shift (morning, afternoon, or evening), as there is no rationale for using dedicated machines. It should be realized that accepting the 'need' for segregation equates to accepting the impossibility of full implementation of basic hygienic precautions, a regrettable situation that entails the risk of transmission of pathogens other than HCV. (KDIGO Hepatitis C guideline 3.1)

\section{Blood Borne Virus Infection (BBV Infection) (Guidelines BBV 5.1-5.8)}

\section{Guideline 5.1 - BBV Infection: Immunisation of patients against hepatitis $B$}

We recommend that patients who require renal replacement therapy (RRT) for CKD should be immunised against HBV. (1A)

\section{Guideline 5.2 - BBV Infection: Immunisation of patients against hepatitis $B$}

We recommend that patients who are likely to require RRT should be identified, whenever possible, early in the course of their disease to receive HBV immunisation. (1A)

\section{Guideline 5.3 - BBV Infection: Immunisation of patients against hepatitis $B$}

We suggest that patients who are at high risk for previous HBV infection should be known to have undetectable anti $\mathrm{HB}$ core antibody (anti $\mathrm{HBc}$ ) before administering an immunisation schedule. (2B)

\section{Guideline 5.4 - BBV Infection: Immunisation of patients against hepatitis $B$}

We recommend that the initial HBV immunisation schedule should involve high doses, frequent doses or both (Fendrix $20 \mu$ g at $0,1,2$ and 6 months; Engerix B $40 \mu \mathrm{g}$ at $0,1,2$ and 6 months; or HBvaxPRO $40 \mu \mathrm{g}$ at 0,1 and 6 months). (1A)

\section{Guideline 5.5 - BBV Infection: Immunisation of patients against hepatitis $B$}

We recommend that the vaccines are licensed for intramuscular route (deltoid muscle) but, if sufficient expertise exists, the intradermal route is more effective. (1A)
Guideline 5.6 - BBV Infection: Immunisation of patients against hepatitis $B$

We recommend that patients should be regarded as a 'responder' if anti HBs antibody titre is $>10 \mathrm{mIU} / \mathrm{mL} 8$ weeks after completing immunisation. (1C)

\section{Guideline 5.7 - BBV Infection: Immunisation of} patients against hepatitis $B$

We recommend that responders to HBV immunisation (as described in 5.6) should receive a further booster dose if the annual anti HBs titre is $<100 \mathrm{mIU} / \mathrm{mL}$. (1B)

\section{Guideline 5.8 - BBV Infection: Immunisation of patients against hepatitis $B$}

We recommend that non-responders to $\mathrm{HBV}$ as described in 5.6 should receive no further immunisation with currently available preparations. (1C)

\section{Blood Borne Virus Infection (BBV Infection) (Guidelines BBV 6.1-6.2)}

\section{Guideline 6.1 - BBV Infection: Immunisation of staff against hepatitis $B$}

We recommend that staff who have clinical contact with patients should be immunised against HBV and demonstrate that they are immune to, or not HBV infective. (1A)

Guideline 6.2 - BBV Infection: Immunisation of staff against hepatitis $B$

We suggest that staff who are not immune to HBV and are not HBV infective should ideally not dialyse patients who are HBV infective. (2B)

\section{Blood Borne Virus Infection (BBV Infection) \\ (Guidelines BBV 7.1-7.4)}

\section{Guideline 7.1 - BBV Infection: HD patients with new $B B V$ infection}

We recommend that, whenever a previously unidentified case of HBV infection is found, units should carry out enhanced HBV surveillance (as described in section 3.8) on all patients who are not immune to HBV (anti HBs titre $>100 \mathrm{mIU} / \mathrm{mL}$ within the last year) who have had a dialysis session since the index patient's last negative test. (1B)

Guideline 7.2 - BBV Infection: HD patients with new $B B V$ infection

We recommend that, whenever a previously unidentified case of HBV infection is found, those patients who 
have anti-HB titre $10-100 \mathrm{mIU} / \mathrm{ml}$ in the preceding 12 months who have had a dialysis session since the index patient's last negative test should also be given a booster dose of Hep B vaccine. Hepatitis B immunoglobulin (HBIG) should be considered for previous non-responders to Hepatitis B vaccine (anti-HBs $<10 \mathrm{mIU} / \mathrm{ml}$ ). (1B)

Guideline 7.3 - BBV Infection: HD patients with new $B B V$ infection

We recommend that, when a previously unidentified case of $\mathrm{HCV}$ is found, enhanced surveillance (as described in section 3.8) should be carried out in all patients who have had a dialysis session since the index patient's last negative test. (1C)

Guideline 7.4-BBV Infection: HD patients with new $B B V$ infection

We recommend that, when a haemodialysis patient develops a new BBV infection, expert virological advice should be obtained to co-ordinate enhanced surveillance of at-risk dialysis patients and carers and to arrange treatment of affected individuals. (1C) 
Summary of Clinical Practice Guideline on Planning, Initiating and Withdrawal of Renal Replacement Therapy

\section{Planning, Initiating and Withdrawal of RRT (Guidelines 1.1-1.2)}

Guideline 1.1 - RRT: Timely nephrology referral We recommend that most patients with CKD stage 4-5 (eGFR $\left.<30 \mathrm{ml} / \mathrm{min} / 1.73 \mathrm{~m}^{2}\right)$ or with CKD stage 3 and rapidly deteriorating renal function should be referred for assessment by a nephrologist. (1B)

Guideline 1.2 - RRT: Timely nephrology referral

We suggest that patients should be referred at least a year before they might be anticipated to require renal replacement therapy (RRT). (2B)

\section{Planning, Initiating and Withdrawal of RRT}

(Guidelines 2.1-2.3)

\section{Guideline 2.1 - RRT: Nephrology follow-up}

We recommend that most patients whose eGFR is $<30 \mathrm{ml} / \mathrm{min} / 1.73 \mathrm{~m}^{2}$ and declining should be under the care of a nephrologist. (1B)

\section{Guideline 2.2 - RRT: Nephrology follow-up}

We recommend that these patients should be managed in a dedicated clinic by a multidisciplinary team. (1B)

\section{Guideline 2.3-RRT: Nephrology follow-up}

We suggest the frequency of nephrology follow-up should be intensified when the eGFR has fallen to $<15 \mathrm{ml} / \mathrm{min} / 1.73 \mathrm{~m}^{2}$ if dialysis treatment is not started. (2D)

\section{Planning, Initiating and Withdrawal of RRT (Guidelines 3.1-3.6)}

\section{Guideline 3.1 - RRT: Preparing patients for RRT}

We recommend that most patients whose eGFR is $<30 \mathrm{ml} / \mathrm{min} / 1.73 \mathrm{~m}^{2}$ and declining should receive timely and personalised information regarding established kidney failure and renal replacement therapy options so they can make an informed decision about treatment. (1B)

Guideline 3.2 - RRT: Preparing patients for RRT

We recommend that patients who present with advanced kidney failure and are likely to need RRT within 3 months should be able to access an accelerated care pathway to deliver education, information and prepare for RRT. (1D)

Guideline 3.3 - RRT: Preparing patients for RRT

We recommend that all medically suitable patients should be informed about the advantages of pre-emptive living kidney transplantation and efforts made to identify a potential donor to allow pre-emptive transplantation before the need for renal replacement therapy. (1B)

\section{Guideline 3.4 - RRT: Preparing patients for RRT}

We recommend that all suitable patients should be listed for cadaveric transplantation six months before the anticipated start of renal replacement therapy. (1B)

\section{Guideline 3.5 - RRT: Preparing patients for RRT}

We suggest that all patients should be encouraged to perform home dialysis therapy where possible, as part of an integrated approach to renal replacement therapy. (2B)

Guideline 3.6 - RRT: Preparing patients for RRT

We suggest that, where home dialysis is not possible, patients and their carers/partners should be actively involved in their dialysis treatment, be encouraged to perform as much self-care as possible and be engaged in all aspects of their treatment including medicines management and changes in diet and lifestyle. (2B)

\section{Planning, Initiating and Withdrawal of RRT (Guidelines 4.1-4.3)}

\section{Guideline 4.1 - RRT: Educating patients and carers for $R R T$}

We recommend that all patients with severe CKD (stage 5 and progressive stage 4), together with their families and carers, should be offered an appropriate education programme aimed at improving their knowledge and understanding of their condition, and of the options for treatment. (1B)

\section{Guideline 4.2 - RRT: Educating patients and carers for $R R T$}

We suggest that education programmes should be multidisciplinary, multifaceted, tailored to the needs of the individual, and based on the principles of adult learning. A variety of approaches should be available including individual conversations, group work, written

Nephron Clin Pract 2011;118(suppl 1):c27-c70 
materials, DVD/CDs, access to expert patients. There should be opportunities for informal follow up. The information imparted should be relevant to the person, with the method, scale, pace and scope of the delivery being suited to the individual's learning style, capacity and preferences. The programme should also include provision for the education of patients who present late, and initiate dialysis in an unplanned fashion. (2C)

\section{Guideline 4.3 - RRT: Educating patients and carers} for RRT

We suggest that pre-renal replacement therapy education programmes for patients and their families and carers should be continued into the treatment phase, with the aims of optimising patient involvement in their own care, improving treatment adherence, and fostering good communication and collaborative relationships with caregivers. (2C)

\section{Planning, Initiating and Withdrawal of RRT (Guidelines 5.1-5.6)}

\section{Guideline 5.1 - RRT: Initiating RRT}

We recommend that patients known to nephrology services for 3 months or more and who are planned to have renal support should start renal replacement therapy in a controlled manner, without the need for hospital admission and using an established access (arteriovenous fistula $[\mathrm{AVF}]$, arteriovenous $[\mathrm{AV}]$ graft, $\mathrm{PD}$ catheter) or by pre-emptive renal transplantation. (1B)

\section{Guideline 5.2 - RRT: Initiating RRT}

We recommend that the decision to start RRT in patients with CKD stage $5\left(\mathrm{eGFR}<15 \mathrm{ml} / \mathrm{min} / 1.73 \mathrm{~m}^{2}\right)$ should be based on a careful discussion with the patient of the risks and benefits of RRT taking into account the patient's symptoms and signs of renal failure, nutritional status, co-morbidity, functional status, and the physical, psychological and social consequences of starting dialysis in that individual. (1D)

\section{Guideline 5.3 - RRT: Initiating RRT}

We suggest that serious consideration should be given to starting renal replacement therapy in patients with an eGFR $<6 \mathrm{ml} / \mathrm{min} / 1.73 \mathrm{~m}^{2}$, even if the patient is asymptomatic. $(2 \mathrm{C})$

\section{Guideline 5.4 - RRT: Initiating RRT}

We recommend that once a decision has been made to start dialysis in a patient with established access there should be no delay in starting treatment i.e. no waiting list to start dialysis. (1D)

\section{Guideline 5.5 - RRT: Initiating RRT}

We recommend that urgent dialysis via a haemodialysis catheter should only be initiated where there is a clear clinical indication. (1D)

\section{Guideline 5.6 - RRT: Initiating RRT}

We suggest that where dialysis is started via an established access (vascular or peritoneal) which fails to work effectively urgent corrective action should be taken and dialysis via a haemodialysis catheter avoided if possible. (2D)

\section{Planning, Initiating and Withdrawal of RRT (Guidelines 6.1-6.5)}

\section{Guideline 6.1 - RRT: End of life care: Conservative kidney management and withdrawal from dialysis}

\section{Conservative kidney care:}

We recommend that patients with advanced kidney disease (CKD stages 4 and 5) who, having opted not to dialyse, are undergoing conservative kidney management and those patients who have imminent or immediate end-of-life care needs should be identified and their care prioritised. (1C)

\section{Guideline 6.2 - RRT: End of life care: Conservative kidney management and withdrawal from dialysis}

Patients who are deteriorating despite dialysis:

We recommend that patients who are struggling to cope on long-term dialysis as a result of progressive deterioration of underlying, irreversible, clinical problems, or sudden onset of a catastrophic clinical event, such as a stroke, should be recognised as being in imminent or immediate need of end-of-life care. (1C)

Guideline 6.3 - RRT: End of life care: Conservative kidney management and withdrawal from dialysis

\section{Advance care planning:}

We recommend that patients with a recognised need for end-of-life care, including those patients undergoing 
conservative kidney care and those deteriorating despite dialysis, should have an Advance Care Plan. (1C)

Guideline 6.4 - RRT: End of life care:

Conservative kidney management and withdrawal from dialysis

\section{Dialysis withdrawal:}

We recommend that any decision to discontinue dialysis should be made within the context of ongoing end-of-life care planning. It should be made jointly by the patient and the responsible nephrologist in full consultation with relatives and carers, the family practitioner and members of the caring team. An assessment of competence should be carried out. Depression may need to be formally excluded. (1C)

Guideline 6.5 - RRT: End of life care: Conservative kidney management and withdrawal from dialysis

\section{Care in the last days:}

We recommend that, in the last days of life, there should be good symptom relief, psychological, spiritual and culturally sensitive care for the dying patient and their family (whether at home, in a hospice or a hospital setting), followed by the provision of culturally appropriate bereavement support. (1C) 


\section{Summary of Clinical Practice Guideline on the Assessment of the Potential Kidney Transplant Recipient}

\section{Access to renal transplantation $(\mathrm{Tx})$ (Guidelines Tx 1.1-1.9)}

Guideline 1.1 - Tx : Access to renal transplantation

We recommend that kidney transplantation should be the renal replacement therapy of choice for the patient with chronic kidney disease stage 5 who is considered fit for major surgery and for chronic immunosuppression. All patients predicted to have an increased life expectancy post-transplantation should be assessed for transplantation. Placement on the transplant waiting list will be limited by individual co-morbidity and prognosis. (1A)

\section{Guideline 1.2 - Tx : Access to renal transplantation}

We recommend that living donor transplantation should be considered the treatment of choice for all patients suitable for renal transplantation when there is an appropriate donor. (1A)

Guideline 1.3 - Tx : Access to renal transplantation We recommend that patients with progressive deterioration in renal function suitable for transplantation should be placed on the national transplant list within six months of their anticipated dialysis start date. Pre-emptive transplantation should be the treatment of choice for all suitable patients whenever a living donor is available. (1A)

Guideline 1.4 - Tx : Access to renal transplantation

We recommend that there must be demonstrable equity of access to deceased donor kidney transplantation irrespective of gender, ethnicity or district of residence. (1A)

Guideline 1.5 - Tx : Access to renal transplantation

We recommend that age is not a contra-indication to transplantation but age related co-morbidity is an important limiting factor. (1B)

Guideline 1.6 - Tx : Access to renal transplantation We recommend that all transplant units should have written criteria for acceptance on to the waiting list. The benefits and potential risks associated with transplantation should be fully explained both verbally and in writing. Potential transplant recipients should be informed of all donor options including living related and unrelated donation and the NHSBT/BTS guidelines for consent for solid organ donation should be followed. (1C)

Guideline 1.7 - Tx : Access to renal transplantation

We recommend that all CKD 5 patients and CKD 4 patients with progressive disease should have their suitability for transplantation assessed annually and that appropriate patients should be referred to a transplant centre. When transplantation is considered inappropriate the reason(s) should be documented. All patients on the transplant list should be assessed annually to determine whether transplantation remains appropriate. Patients should be placed on, or removed from the waiting list only after discussion and agreement with the nephrologist, transplant surgeon and the patients themselves according to local practice. (1C)

Guideline 1.8 - Tx : Access to renal transplantation

We recommend that the care of the renal transplant recipient is best undertaken by a multi-disciplinary team. (1C)

Guideline 1.9 - Tx : Access to renal transplantation

We recommend that simultaneous kidney-pancreas transplantation or living donor renal transplantation is the treatment of choice for patients with Type 1 diabetes mellitus who are suitable for renal transplantation. (1B)

2. Evaluation, selection and preparation of the potential transplant recipient ( $\mathrm{Tx}$ ) (Guidelines $\mathrm{Tx}$ 2.1-2.9)

\section{Guideline 2.1 - Tx : Pre-transplant assessment}

We recommend that the object of pre-transplant assessment is: (a) to ensure transplantation is technically possible; (b) to ensure the recipient's chances of survival are not compromised by transplantation; (c) to ensure that graft survival is not limited by premature death (maximum benefit obtained from a limited resource); (d) to ensure pre-existing conditions are not exacerbated by transplantation; (e) to identify measures to be taken to minimise peri- and post-operative complications; (f) to inform patients of the likely risks and benefits of transplantation. (1C)

Guideline 2.2 - Tx : Pre-transplant cardiac assessment

We suggest that there is no compelling evidence that pre-transplantation screening tests for coronary artery 
disease in asymptomatic patients with established renal failure is effective in preventing future cardiac events or reducing mortality after transplantation. Until better evidence emerges, screening tests may be best used to identify high-risk patients for exclusion from the transplant waiting list. (2C)

\section{Guideline 2.3 - Tx : Preparation of the renal transplant recipient}

We suggest that the use of pre-operative beta-blockers may be considered in patients at high cardiovascular risk undergoing renal transplantation but should be introduced at least 1 month before transplantation. Beta-blockers should not be discontinued abruptly peri-operatively. Low dose aspirin and clopidogrel therapy are not contraindications to transplantation. (2C)

\section{Guideline 2.4 - Tx : Preparation of the renal transplant recipient}

We recommend that patients should be strongly encouraged to stop smoking before and after transplantation. Formal smoking cessation programmes should be offered and accessed in primary care. (1A)

\section{Guideline 2.5 - Tx : Preparation of the renal transplant recipient}

We suggest that obese patients (BMI $>30 \mathrm{~kg} / \mathrm{m}^{2}$ ) present technical difficulties and are at increased risk of peri-operative complications. They should be screened rigorously for cardiovascular disease and each case considered individually. Although obesity is not an absolute contra-indication to transplantation, individuals with a BMI $>40 \mathrm{~kg} / \mathrm{m}^{2}$ are less likely to benefit. (2B)

\section{Guideline 2.6 - Tx : Preparation of the renal transplant recipient}

We recommend that all potential transplant recipients should be tested for prior exposure to viral infections including: cytomegalovirus (CMV), Epstein-Barr virus (EBV), varicella zoster virus (VZV), hepatitis B and C and human immunodeficiency virus (HIV). Immunisation should be offered to all hepatitis B (if not already immunised) and VZ virus antibody negative patients before transplantation. Patients otherwise suitable for renal transplantation with evidence of chronic hepatitis B and/or C or HIV infection should be managed according to British Transplantation Society and European Best Practice Guidelines prior to transplantation. (1A)

\section{Guideline 2.7 - Tx : Evaluation and selection of the renal transplant recipient}

We recommend that renal transplantation should only be considered in potential recipients with previous malignancy (excluding non-melanoma skin cancer) if there is no evidence of persistent cancer. It is recommended that the waiting time between successful tumour treatment/remission and transplantation be at least 2 years. For certain malignancies the waiting time may need to be extended to more than 5 years. The Israel Penn International Transplant Tumour Registry should be consulted for tumour specific advice (www. ipittr.uc.edu/Home.cfm). (1A)

\section{Guideline 2.8 - Tx : Evaluation and selection of the} renal transplant recipient

We recommend that patients who are at risk of developing recurrence of original renal disease should be managed according to the European Best Practice Guidelines (EBPG). (not graded)

\section{Guideline 2.9-Tx : Screening investigations in the renal transplant recipient}

We suggest that there is no evidence that asymptomatic potential transplant recipients require screening for diverticular disease, peptic ulceration or gall bladder stones. (2C) 


\section{Summary of Clinical Practice Guideline on Vascular Access for Haemodialysis}

\section{Preferred type of vascular access (Guidelines 1.1-1.3)}

\section{Guideline 1.1 - Incident patient vascular access}

We recommend that any individual who commences haemodialysis should do so with an arteriovenous fistula as first choice, an arteriovenous graft as second choice, a tunnelled venous catheter as third choice and a non tunnelled catheter as an option of necessity. (1B)

\section{Guideline 1.2 - Prevalent patient vascular access}

We suggest that any patients on long term haemodialysis should have vascular access monitored and maintained to minimise failure to allow timely planning for subsequent replacement of optimal vascular (or PD) access and avoid the need for emergency access. (2B)

\section{Guideline 1.3 - Complications related to vascular access}

We recommend that any patients on long term haemodialysis should have the risk of complications, especially infection, related to vascular access minimised by appropriate interventions. (1B)

2. Preservation of sites for native vascular access (Guidelines 2.1-2.2)

Guideline 2.1 - Preservation of peripheral veins for vascular access

We suggest that all patients that may require renal replacement therapy should have education on forearm vein preservation. (2D)

Guideline 2.2 - Preservation of peripheral veins for vascular access

We suggest that healthcare workers should avoid unnecessary venepunctures and peripheral venous access in the upper limb intended for creation of vascular access. (2C)

3. Timing of creation of vascular access (Guidelines 3.1-3.2)

Guideline 3.1 - Planning of vascular access

We suggest that planning for access should commence when patients enter CKD stage 4 . (2C)
Guideline 3.2 - Creation of vascular access

We recommend that the exact timing of placement of vascular access will be determined by rate of decline of renal function, co-morbidities and by the surgical pathway. (1C)

\section{Maintenance of vascular access (Guidelines} 4.1-4.4)

\section{Guideline 4.1 - Pharmacological treatment}

We suggest that there are no proven long term pharmacological interventions that have shown to improve access survival. (2C)

\section{Guideline 4.2 - Needling technique}

We suggest that buttonhole is the preferred needling technique. (2B)

\section{Guideline 4.3 - Vascular access surveillance}

We suggest that systematic observation and advanced surveillance should be employed to predict and prevent access failure. (2C)

\section{Guideline 4.4 - Intervention for failing vascular} access

We recommend that a local standard operating policy for intervention should be developed. (1C)

\section{Prevention of catheter related infections} (Guidelines 5.1-5.4)

\section{Guideline 5.1 - Minimise the use of venous catheters}

We recommend that venous catheters should be employed as a method of last resort for longer term vascular access to reduce the overall risk of infectious complications in haemodialysis patients. (1B)

\section{Guideline 5.2-Minimising the risk of catheter related infection}

We suggest that aseptic technique should be mandatory at every manipulation of central venous dialysis catheters. (2C)

\section{Guideline 5.3 - Minimising the risk of catheter related infection}

We recommend that the catheter exit site should be cleaned with Chlorhexidine 2\%. (1B) 
Guideline 5.4-Minimising the risk of catheter related infection

We suggest that an antimicrobial or antibiotic lock solution be used to reduce catheter related bacteraemia and other infections. (2B)

\section{Complications of vascular access (Guidelines 6.1-6.4)}

\section{Guideline 6.1 - Treatment of access infection and} related bacteraemia

We recommend that venous catheters should be removed in all seriously ill haemodialysis patients with catheter related bacteraemia unless no alternative vascular access can be achieved. (1B)

\section{Guideline 6.2 - Prevention of arteriovenous aneurysmal formation}

We suggest that prevention of aneurysmal formation with good needling technique is appropriate and is the cornerstone for preserving arteriovenous fistulae. (2C)
Guideline 6.3 - Treatment of ischemia related to arteriovenous fistulae or grafts

We suggest that the development of peripheral ischaemia related to arteriovenous fistulae or grafts requires early review by the vascular access surgeon to allow proactive intervention to prevent the onset of gangrene or need for amputation. (2B)

Guideline 6.4 - Prevention and treatment of central venous catheter occlusion

We suggest that catheter occlusion may be prevented by the use of an antithrombotic lock solution and catheter occlusion should be managed by using thrombolytic agents (urokinase or t-PA) before catheter guidewire exchange or replacement. (2C) 


\section{Summary of Clinical Practice Guideline on Haemodialysis}

\section{Haemodialysis (HD) (Guidelines 1.1-1.6)}

\section{Guideline 1.1 - HD: Haemodialysis facilities}

We recommend that the specification of new or refurbished HD facilities should adhere to the guidelines that are described in the NHS Estates Health Building Notes 07-01 Satellite Dialysis Unit and 07-02 Main Renal Unit. (1C)

\section{Guideline 1.2 - HD: Haemodialysis facilities}

We recommend that the HD facility should have sufficient specialist support staff to fulfil the criteria listed by the Renal Workforce Planning Group 2002. (1C)

\section{Guideline 1.3 - HD: Haemodialysis facilities}

We recommend that, except in remote geographical areas the travel time to a HD facility should be less than 30 minutes or a HD facility should be located with 25 miles of the patient's home. In inner city areas travel times over short distances may exceed 30 minutes at peak traffic flow periods during the day. (1B)

\section{Guideline 1.4 - HD: Haemodialysis facilities}

We suggest that HD patients who require transport should be collected from home within 30 minutes of the allotted time and be collected to return home within 30 minutes of finishing dialysis. (2C)

\section{Guideline 1.5 - HD: Haemodialysis facilities}

We suggest that HD capacity in satellite and main renal units within a geographical area should increase in step with predicted need. The national average number of hospital HD patients per million catchment population reported for the previous year by the UK Renal Registry should be regarded as the minimum capacity for $\mathrm{HD}$ in each geographically based renal service. (2C)

\section{Guideline 1.6 - HD: Haemodialysis facilities}

We suggest that the required number of HD stations should be based on using each station for 2 patients per day three times per week. This approach allows for patient choice regarding HD schedules, more frequent dialysis schedules, provision of holiday HD and expansion in patient numbers. $(2 \mathrm{C})$

\section{Haemodialysis (HD) (Guidelines 2.1-2.3)}

Guideline 2.1 - HD: Haemodialysis equipment and disposables

We recommend that all equipment used in the delivery and monitoring of HD should be CE marked and approved to ensure compliance with the relevant safety standards BS EN 60601-1:2006 General safety standards for electrical equipment in clinical use (currently under revision with the revised version being available in 2010) and BS EN 60601-2-16:2008 Particular requirements for basic safety and essential performance of haemodialysis, haemodiafiltration and haemofiltration equipment. (1C)

\section{Guideline 2.2 - HD: Haemodialysis equipment and} disposables

We recommend that all disposable items used in the delivery of HD (such as haemodialysers, associated devices and the extracorporeal circuits used with such devices) should be CE marked to indicate compliance with the relevant standards. (1C)

\section{Guideline 2.3 - HD: Haemodialysis equipment and disposables}

We suggest that machines should be replaced after between seven and ten years' service or after completing between 25,000 and 40,000 hours of use for HD, depending upon an assessment of machine condition. (2C)

\section{Haemodialysis (HD) (Guidelines 3.1-3.8)}

Guideline 3.1 - HD: Concentrates for haemodialysis

We recommend that commercially produced concentrates are classified as medical devices and should be CE marked to demonstrate compliance with BS ISO 13958:2009 Concentrates for haemodialysis and related therapies and the water used for diluting the concentrates should comply with BS ISO 13959:2009 Water for haemodialysis and related therapies or meet the requirements stated in the European Pharmacopoeia (6th edition, 2007). (1C)

Guideline 3.2 - HD: Specification of water treatment system for haemodialysis

We recommend that the complete water treatment, storage and distribution system should meet the requirements of BS ISO 26722:2009 Water treatment equipment for haemodialysis applications and related therapies and be shown to be capable of meeting the requirements of BS 
ISO 13959:2009 Water for haemodialysis and related therapies at the time of installation. (1C)

Guideline 3.3 - HD: Chemical contaminants in water used for the preparation of dialysis fluid

We recommend that the concentrations of chemical contaminants in water used to prepare dialysis fluid should not exceed the limits stated either in BS ISO 13959:2009 Water for haemodialysis and related therapies or in the European Pharmacopoeia (6th edition, 2007). A programme of improvement should begin immediately if routine monitoring demonstrates that concentrations of chemical contaminants exceed the maximum allowable limits. (1B)

\section{Guideline 3.4 - HD: Microbiological contaminants} in water used for the preparation of dialysis fluid

We recommend that the concentration of microbiological contaminants in water used for the preparation of the dialysis fluid should not exceed the limits stated in BS ISO 11663:2009 Quality of dialysis fluid for haemodialysis and related therapies. (1C)

\section{Guideline 3.5 - HD: Microbiological contaminants in dialysis fluid}

We recommend that dialysis fluid is produced by the mixing of treated water, acid and bicarbonate concentrates. The microbiological contaminant levels for acid and bicarbonate concentrates are defined in BS ISO 13958:2009 Concentrates for haemodialysis and related therapies. The microbiological quality of the dialysis fluid should not exceed the limits specified in BS ISO 11663:2009 Quality of dialysis fluid for haemodialysis and related therapies (100 CFU/ml for bacteria and $0.25 \mathrm{EU} / \mathrm{ml}$ for endotoxin). If routine monitoring demonstrates microbiological contaminant levels in excess of $50 \mathrm{CFU} / \mathrm{ml}$ and $0.125 \mathrm{EU} / \mathrm{ml}$ for bacteria and endotoxin (50\% of the maximum permitted levels) a programme of corrective measures should be commenced immediately. (1B)

\section{Guideline 3.6 - HD: Ultrapure dialysis fluid}

We recommend that all new water treatment plants should be capable of producing water suitable for the production of 'ultrapure dialysis fluid'. The microbiological contaminant levels of ultrapure dialysis fluid should be $<0.1 \mathrm{CFU} / \mathrm{mL}$ and $<0.03 \mathrm{EU} / \mathrm{mL}$. (1B)

\section{Guideline 3.7 - HD: Monitoring of feed and dialysis water for haemodialysis}

We recommend that a routine testing procedure for water for dialysis should form part of the renal unit policy. Each unit should have standard operating procedures in place for sampling, monitoring and recording of feed and product water quality. The operating procedures should include details of the procedures to be followed if the prescribed limits are exceeded. (1C)

\section{Guideline 3.8 - HD: Bicarbonate dialysate for haemodialysis}

We recommend that the dialysate should contain bicarbonate as the buffer. (1B)

\section{Haemodialysis (HD) (Guidelines 4.1-4.5)}

\section{Guideline 4.1 - HD: Biocompatible haemodialysis}

We suggest that haemodialysers with synthetic and modified cellulose membranes should be used instead of unmodified cellulose membranes. The proven benefits of low flux synthetic and modified cellulose membranes over unmodified cellulose membranes are limited to advantages arising from different aspects of improved biocompatibility rather than better patient outcomes. (2C)

\section{Guideline 4.2 - HD: High flux HD membranes}

We suggest that high flux dialysers should be used instead of low flux dialysers to provide HD. Evidence of improved patient survival with the use of high flux membranes is restricted to incident patients, who have lower serum albumin concentrations $(<40 \mathrm{~g} / \mathrm{L})$ or have diabetes mellitus, and prevalent patients, who have been on $\mathrm{HD}$ for more than 3.7 years. (2B)

\section{Guideline 4.3 - HD: High flux HD and haemodiafiltration}

Both modalities are effective extracorporeal techniques for established renal failure but haemodiafiltration can provide higher rates of removal of small and middle molecules and may lower the risk of developing complications due to dialysis related amyloidosis. Haemodiafiltration would be the preferred mode of extracorporeal renal replacement therapy in patients with established renal failure if it was shown in randomised controlled trials to provide better patient outcomes than high flux HD. We suggest that high flux HD using ultrapure water provides non-inferior patient outcomes to haemodiafiltration. (2C)

\section{Guideline 4.4 - HD: Haemodialysis membranes}

We recommend that the use of dialysers sterilised with ethylene oxide should be avoided. (1C) 
Guideline 4.5 - HD: Haemodialysis membranes

We recommend that ACE inhibitor drugs should not be prescribed in patients who are receiving $\mathrm{HD}$ with synthetic membranes which are capable of generating bradykinin. (1B)

\section{Haemodialysis (HD) (Guidelines 5.1-5.10)}

\section{Guideline 5.1 - HD: Minimum frequency of haemodialysis per week}

We recommend that HD should take place at least three times per week in nearly all patients with established renal failure. Reduction of dialysis frequency to twice per week because of insufficient dialysis facilities is unacceptable. (1B)

\section{Guideline 5.2 - HD: Method of measuring haemodialysis dose}

We recommend that a standard method of measuring dialysis dose is adopted to permit effective comparative audit within each regional network and national registry. (1B)

\section{Guideline 5.3 - HD: Minimum dose of thrice weekly} haemodialysis

We recommend that every patient with end-stage chronic renal failure receiving thrice weekly HD should have consistently:

- either urea reduction ratio (URR) $>65 \%$

- or equilibrated Kt/V of $>1.2$ (or sp Kt/V of $>1.3$ ) calculated from pre- and post-dialysis urea values, duration of dialysis and weight loss during dialysis.

To achieve a URR above $65 \%$ or eKt/V above 1.2 consistently in the vast majority of the haemodialysis population clinicians should aim for a minimum target URR of $70 \%$ or minimum eKt/V of 1.3 in individual patients. Aiming for these target doses also addresses the concerns raised by recent data which suggest that women and patients of low body weight may have improved survival rates if the URR is maintained above $70 \%$ or $\mathrm{eKt} / \mathrm{V}$ is at least 1.3 . (1A)

\section{Guideline 5.4 - HD: Minimum duration of thrice weekly haemodialysis}

We recommend that the duration of thrice weekly HD in adult patients with minimal residual renal function should not be reduced below 4 hours without careful consideration. (1B)
Guideline 5.5 - HD: Weekly haemodialysis dose

We suggest that adequate HD three times per week should be defined as a combination of the minimum recommended dialysis dose (URR $>65 \%$ or $\mathrm{eKt} / \mathrm{V}$ $>1.2$ ) and a minimum recommended treatment time per session (240 mins). (2C)

\section{Guideline 5.6 - HD: Increased frequency and/or duration of haemodialysis}

We suggest that an increase in treatment and/or frequency of HD should be considered in patients with refractory fluid overload, uncontrolled hypertension, hyperphosphataemia, malnutrition or cardiovascular disease. (2C)

\section{Guideline 5.7 - HD: Frequency of monitoring haemodialysis dose}

We suggest that the measurement of the 'dose' or 'adequacy' of HD should be performed monthly in all hospital HD patients and may be performed less frequently in home HD patients. All dialysis units should collect and report this data to their regional network and the UK Renal Registry. (1C)

\section{Guideline 5.8 - HD: Haemodialysis post-dialysis blood sampling}

We recommend the use of a standardised method of post-dialysis blood sampling. Post-dialysis blood samples should be collected by the stop-dialysate flow method or, alternatively, the slow-flow or the simplified stop-flow methods may be used. The method used should remain consistent within renal units and should be reported to the Renal Registry. (1B)

\section{Guideline 5.9 - HD: Residual renal function}

We suggest that the management of haemodialysis patients should include dialysis strategies that attempt to preserve their residual renal function. As a minimum policies and procedures should be in place to reduce intradialysis hypotension or excessive ultrafiltration and avoid the use of nephrotoxins. (2B)

\section{Guideline 5.10 - HD: Haemodialysis frequency and} dose in acute kidney injury

We suggest that patients with acute kidney injury and multi-organ failure, who are treated by intermittent HD, should receive alternate day HD at a dose at least equal to the minimum dose for established renal failure (URR $>65 \%$ or eKt/V >1.2) or daily HD. Haemodynamically unstable patients with AKI may be treated 
by a continuous modality of RRT and the minimum recommended dose of continuous renal replacement therapy is $20 \mathrm{ml} / \mathrm{kg} /$ hour. (2C)

\section{Haemodialysis (HD) (Guidelines 6.1-6.8)}

\section{Guideline 6.1 - HD: Standardisation of the method} of pre-dialysis blood sampling

We recommend that blood sampling for biochemical and haematological measurements should be performed before a mid-week HD session using a dry needle or syringe. (1C)

\section{Guideline 6.2 - HD: Frequency of monitoring biochemical and haematological indices}

We recommend that monitoring of pre-dialysis biochemical and haematological parameters should be performed monthly in hospital HD patients and at least 3 monthly in home HD patients. (1D)

\section{Guideline 6.3 - HD: Pre-dialysis serum bicarbonate} concentrations

We suggest that pre-dialysis serum bicarbonate concentrations measured with minimum delay after venepuncture should be between 18 and $24 \mathrm{mmol} / \mathrm{l}$. (2C)

\section{Guideline 6.4 - HD: Pre-dialysis serum potassium concentrations}

We suggest that pre-dialysis serum potassium should be between 4.0 and $6.0 \mathrm{mmol} / \mathrm{l}$ in HD patients. (2C)

\section{Guideline 6.5 - HD: Pre-dialysis serum phosphate concentrations}

We suggest that pre-dialysis serum phosphate, if elevated, should be lowered towards the normal range, such as between 1.1 and $1.7 \mathrm{mmol} / \mathrm{l}$. (2C)

\section{Guideline 6.6 - HD: Pre-dialysis serum calcium concentrations}

We suggest that pre-dialysis serum calcium, adjusted for serum albumin, should be within the normal range. $(2 \mathrm{C})$

\section{Guideline 6.7 - HD: Serum aluminium concentrations}

We suggest that serum aluminium concentration should be measured every three months in all patients receiving oral aluminium hydroxide. No patient whose ferritin level is $<100 \mu \mathrm{g} / \mathrm{l}$ should have a serum aluminium concentration $>60 \mu \mathrm{g} / \mathrm{l}(2.2 \mu \mathrm{mol} / \mathrm{l})$. (2C)
Guideline 6.8 - HD: Pre-dialysis haemoglobin concentrations

We recommend that pre-dialysis haemoglobin concentration should be maintained within the range 10 $12 \mathrm{~g} / \mathrm{dl}$. (1B)

\section{Haemodialysis (HD) (Guidelines 7.1-7.4)}

Guideline 7.1 - HD: Anticoagulation without added risk of bleeding

We recommend that patients without increased bleeding risk should be given unfractionated heparin or LMWH during HD to reduce the risk of clotting of the extracorporeal system. (1A)

Guideline 7.2-HD: Anticoagulation with significant risk of bleeding

We recommend that anticoagulation should be avoided or kept to a minimum in patients with a high risk of bleeding. This can be achieved by using a high blood flow rate and regular flushing of the extracorporeal circuit with saline every 15-30 minutes or regional citrate infusion. (1C)

\section{Guideline 7.3 - HD: Anticoagulation in patients with} HIT type 2 or HITTS

We suggest that patients with HIT type 2 or HITTS should not be prescribed unfractionated heparin or low molecular weight heparin (LMWH). (2B)

\section{Guideline 7.4 - HD: Anticoagulation and catheter lock solutions}

We suggest that each unit should have policies and procedures for administration of catheter locking solutions to maintain catheter patency and keep systemic leak of the catheter lock solution to a minimum. (2C)

\section{Haemodialysis (HD) (Guidelines 8.1-8.4)}

\section{Guideline 8.1 - HD: Symptomatic dialysis-related hypotension}

We recommend that data on the frequency of dialysisrelated hypotension, defined as an acute symptomatic fall in blood pressure during dialysis requiring immediate intervention to prevent syncope, should be collected and audited. (1C)

\section{Guideline 8.2 - HD: Prevention of symptomatic} dialysis-related hypotension

We recommend that a stepwise approach is adopted to try and reduce the incidence of intradialysis hypotension:

Nephron Clin Pract 2011;118(suppl 1):c27-c70 
restrict dietary sodium intake and review 'dry weight' and antihypertensive drugs; increase duration of HD to reduce the hourly ultrafiltration rate; trial use of cool temperature dialysis. (2C)

Guideline 8.3 - HD: Maximum hourly ultrafiltration rate

We suggest that the maximum hourly ultrafiltration rate during haemodialysis should not exceed $10 \mathrm{ml} / \mathrm{kg} /$ hour. (2C)

\section{Guideline 8.4 - HD: Prevention and detection of} venous fistula needle or venous line disconnection

We suggest that all haemodialysis staff should follow standard operating procedures to minimise the risk of accidental venous needle/line disconnection. In patients who are restless or undergoing haemodialysis at home consideration should be given to the use of commercially available monitoring systems. (2C)

\section{Haemodialysis (HD) (Guidelines 9.1-9.3)}

Guideline 9.1 - HD: Home haemodialysis and patient choice

We recommend that all patients who may be suitable for home dialysis should receive full information and education about home HD. (1B)

\section{Guideline 9.2 - HD: Home haemodialysis training} and technical support

We suggest that patients may need to travel to a sub-regional or regional centre to pursue their choice to train for home HD if home HD training is not available locally. (2C)

\section{Guideline 9.3 - HD: Daily home haemodialysis}

We recommend self-treatment at home as the best way to perform daily short or daily nocturnal HD. (1D) 


\section{Summary of Clinical Practice Guidelines on Peritoneal Dialysis}

\section{Peritoneal Dialysis (PD) (Guidelines PD 1.1-1.5)}

\section{Guideline 1.1 - PD: Equipment and Resources}

Guideline 1.1.1 - PD: Resources

We recommend that Peritoneal Dialysis should be delivered in the context of a comprehensive and integrated service for renal replacement therapies, including haemodialysis (including temporary backup facilities), transplantation and conservative care. Both continuous ambulatory peritoneal dialysis (CAPD) and automated peritoneal dialysis (APD), in all its forms should be available. (1C)

\section{Guideline 1.1.2 - PD: Resources}

We recommend that dedicated PD nursing staff (1 W.T.E. per 20 patients) should be part of the multidisciplinary team. (1C)

\section{Guideline 1.1.3 - PD: Resources}

We recommend that each unit has a designated lead clinician for PD. (1C)

\section{Guideline 1.1.4 - PD: Resources}

We recommend that assisted PD should be available to patients wishing to have home dialysis treatment but unable to perform self-care PD. (1C)

\section{Guideline 1.2 - PD: Equipment and Resources}

We recommend that all equipment used in the delivery and monitoring of therapies should comply with the relevant standards for medical electrical equipment [BS-EN 60601-2-39:1999, BS5724-2-39:1998, IEC 60601-2-39:1998, Particular requirements for the safety - specification for peritoneal dialysis equipment]. Tubing sets and catheters should carry the 'CE' mark to indicate that the item conforms to the essential requirements of the Medical Devices Directive (93/42/ EEC) and that its conformity has been assessed in accordance with the directive. (1C)

\section{Guideline 1.3 - PD: Equipment and Resources}

We recommend that fluids for peritoneal dialysis are required to satisfy the current European quality standards as indicated in the European good manufacturing practice and the European Pharmacopoeia Monograph 'Solutions for Peritoneal Dialysis'. Manufacturing facilities are required to meet the relevant standards
(ISO 9001/2 and EN 46001/2). Product registration files must be submitted to and product approval given by the Medicines Control Agency. (1C)

\section{Guideline 1.4 - PD: Equipment and Resources}

We recommend that the use of disconnect systems should be standard unless clinically contraindicated. (1A)

\section{Guideline 1.5 - PD: Equipment and Resources}

We suggest that biocompatible PD solutions (normal $\mathrm{pH}$, low concentrations of glucose degradation products) should be used in patients experiencing infusion pain. (2B)

\section{Peritoneal Dialysis (PD) (Guidelines PD 2.1-2.4)}

\section{Guideline 2.1 - PD: Preparation for Peritoneal Dialysis}

We recommend that all patients should, where possible, be adequately prepared for renal replacement therapy and this should include receiving information and education about PD treatment, delivered by an experienced member of the MDT. Patients commencing RRT in an unplanned fashion for whatever reason should receive this information once appropriate. (1C) Fast track education and urgent PD catheter insertion with acute start of PD should be available, and be offered to suitable patients urgently starting on RRT who wish to avoid temporary haemodialysis. (1C)

\section{Guideline 2.2 - PD: Preparation for Peritoneal Dialysis}

We recommend that, where possible, timing of PD catheter insertion should be planned to accommodate patient convenience, and commencement of training between 10 days and 6 weeks before RRT is essential to enable correction of early catheter-related problems without the need for temporary haemodialysis. (1C)

\section{Guideline 2.3 - PD: Preparation for Peritoneal Dialysis}

We recommend that $\mathrm{PD}$ catheter insertion practice should be managed according to the Renal Association Peritoneal Access Guidelines.

\section{Guideline 2.4 - PD: Preparation for Peritoneal Dialysis}

We recommend that peri-operative catheter care and catheter complications (leaks, hernias, obstruction) should be managed according to the International Society of Peritoneal Dialysis guidelines. 
3. Peritoneal Dialysis (PD) (Guidelines PD 3.1-3.3)

\section{Guideline 3.1 - PD: Solute Clearance}

We recommend that both residual urine and peritoneal dialysis components of small solute clearance should be measured at least six monthly or more frequently if dependant on residual renal function to achieve clearance targets or if clinically or biochemically indicated. Both urea and/or creatinine clearances can be used to monitor dialysis adequacy and should be interpreted within the limits of the methods. (1C)

\section{Guideline 3.2.1 - PD: Solute Clearance}

We recommend that a combined urinary and peritoneal $\mathrm{Kt} / \mathrm{V}_{\text {urea }}$ of $1.7 /$ week or a creatinine clearance of $50 \mathrm{~L} /$ week $/ 1.73 \mathrm{~m}^{2}$ should be considered as minimal treatment doses. (1A)

\section{Guideline 3.2.2 - PD: Solute Clearance}

We recommend that the dose should be increased in patients experiencing uraemic symptoms. (1B)

\section{Guideline 3.3 - PD: Solute Clearance}

We recommend that a continuous 24 hour PD regime is preferred to an intermittent regime. (1B)

\section{Peritoneal Dialysis (PD) (Guidelines PD 4.1-4.5)}

\section{Guideline 4.1 - PD: Ultrafiltration and Fluid Management}

We recommend that peritoneal membrane function should be monitored regularly (6 weeks after commencing treatment and at least annually or when clinically indicated) using a peritoneal equilibration test (PET) or equivalent. Daily urine and peritoneal ultrafiltration volumes, with appropriate correction for overfill, should be monitored at least six-monthly. (1C)

\section{Guideline 4.2 - PD: Ultrafiltration and Fluid Management}

We recommend that dialysis regimens resulting in fluid reabsorption should be avoided. Patients with high or high average solute transport, at greatest risk of this problem, should be considered for APD and icodextrin. (1A)

\section{Guideline 4.3 - PD: Ultrafiltration and Fluid Management}

We recommend that dialysis regimens resulting in routine utilisation of hypertonic (3.86\%) glucose exchanges should be avoided. Where appropriate this should be achieved by using icodextrin or diuretics. (1B)

\section{Guideline 4.4 - PD: Ultrafiltration and Fluid Management}

We recommend that treatment strategies that favour preservation of renal function should be adopted where possible. These include the use of ACEi, ARBs and diuretics, and the avoidance of episodes of dehydration. (1B)

\section{Guideline 4.5 - PD: Ultrafiltration and Fluid Management}

We recommend that anuric patients who consistently achieve a daily ultrafiltration of less than $750 \mathrm{ml}$ should be closely monitored and the benefits of modality switch considered. (1B)

\section{Peritoneal Dialysis (PD) (Guidelines PD 5.1-5.2)}

\section{Guideline 5.1 - PD: Infectious Complications}

Guideline 5.1.1 - PD Infectious Complications:

\section{Prevention Strategies}

We recommend that PD units should undertake regular audit of their peritonitis and exit-site infection rates, including causative organism, treatment and outcomes. They should enter into active dialogue with their microbiology department and infection control team to develop optimal local treatment and prevention protocols. (1B)

\section{Guideline 5.1.2 - PD Infectious Complications: Prevention Strategies}

We recommend that flush-before-fill dialysis delivery systems should be used. (1A)

\section{Guideline 5.1.3 - PD Infectious Complications: Prevention Strategies}

We recommend that patients should undergo regular revision of their technique (at least annually or more frequently if indicated, such as after an episode of PDrelated infection or a significant interruption to the patient performing PD) and receive intensified training if this is below standard. (1C)

\section{Guideline 5.1.4 - PD Infectious Complications: Prevention Strategies}

We recommend that initial catheter insertion should be accompanied by antibiotic prophylaxis. (1B) 
Guideline 5.1.5 - PD Infectious Complications: Prevention Strategies

We recommend that invasive procedures should be accompanied by antibiotic prophylaxis and emptying the abdomen of dialysis fluid for a period commensurate with the procedure. (1C)

\section{Guideline 5.1.6 - PD Infectious Complications: Prevention Strategies}

We recommend that topical antibiotic administration should be used to reduce the frequency of Staph. aureus and Gram negative exit-site infection and peritonitis. (1A)

\section{Guideline 5.2 - PD Infectious Complications Guideline 5.2.1 - PD Infectious Complications: Treatment}

We recommend that exit site infection is suggested by pain, swelling, crusting, erythema and serous discharge; purulent discharge always indicates infection. Swabs should be taken for culture and initial empiric therapy should be with oral antibiotics that will cover $S$. aureus and $P$. aeruginosa. (1B)

\section{Guideline 5.2.2 - PD Infectious Complications: Treatment}

We recommend that methicillin resistant organisms (MRSA) will require systemic treatment (e.g. vancomycin) and will need to comply with local infection control policies. (1C)

\section{Guideline 5.2.3 - PD Infectious Complications: Treatment}

We recommend that initial treatment regimens for peritonitis should include cover for bacterial Gram positive and Gram negative organisms including Pseudomonas species until result of culture and antibiotic sensitivities are obtained. (1C)

\section{Peritoneal Dialysis (PD) (Guidelines PD 6.1-6.4)}

\section{Guideline 6.1 - PD: Metabolic Factors}

We recommend that standard strategies to optimise diabetic control should be used; these should be complemented by dialysis prescription regimens that minimise glucose, including glucose free solutions (icodextrin and amino-acids), where possible. (1B)

\section{Guideline 6.2 - PD: Metabolic Factors}

We recommend that plasma bicarbonate should be maintained within the normal range; this can be achieved in the vast majority of patients by adjusting the dialysis dose and/or dialysate buffer concentration. Occasionally bicarbonate buffered solutions will be required. (1B)

\section{Guideline 6.3 - PD: Metabolic Factors}

We suggest that central obesity can worsen or develop in some PD patients. The risk of this problem, and associated metabolic complications, notably increased atherogenicity of lipid profiles and insulin resistance, can be reduced by avoiding excessive glucose prescription and using icodextrin. (2C)

\section{Guideline 6.4 - PD: Metabolic Factors}

We recommend that awareness of the effects of icodextrin on assays for estimation of amylase and glucose (using glucose dehydrogenase) should be disseminated to patients, relatives, laboratory and clinical staff. (1C)

\section{Peritoneal Dialysis (PD) (Guidelines PD 7.1)}

\section{Guideline 7.1 - PD: Encapsulating Peritoneal Sclerosis}

We recommend that the diagnosis and management of encapsulating peritoneal sclerosis (EPS), including consideration of surgical management of EPS, should follow the principles outlined in the UK Encapsulating Peritoneal Sclerosis Clinical Practice Guidelines. (not graded)

\section{Guideline 7.2 - PD: Encapsulating Peritoneal Sclerosis}

We recommend that there is no optimal duration of peritoneal dialysis and decisions regarding the duration of therapy should be tailored to the individual patient, taking into account clinical and social factors and patients wishes, and should follow the principles outlined in the ISPD Length of Time on Peritoneal Dialysis and Encapsulating Peritoneal Sclerosis Position Paper. (not graded) 
Summary of Clinical Practice Guideline on Peritoneal Dialysis Access

\section{Peritoneal Dialysis Access (PD Access)}

(Guideline 1.1)

\section{Guideline 1.1 - PD Access: Access Team}

We recommend that each centre should have a dedicated team involved in the implantation and care of peritoneal catheters. (1C)

\section{Peritoneal Dialysis Access (PD Access)}

(Guideline 2.1)

\section{Guideline 2.1 - PD Access: Timing}

We suggest, whenever possible, that catheter insertion should be performed at least 2 weeks before starting peritoneal dialysis. Small dialysate volumes in the supine position can be used if dialysis is required earlier. (2B)

\section{Peritoneal Dialysis Access (PD Access)}

(Guideline 3.1)

\section{Guideline 3.1 - PD Access: Implantation Protocol}

We recommend that renal units should have clear protocols for peri-operative catheter care including the use of antibiotic prophylaxis. (1A)

\section{Peritoneal Dialysis Access (PD Access) \\ (Guidelines 4.1-4.4)}

Guideline 4.1 - PD Access: Implantation Technique

We recommend that local expertise at individual centres should govern the choice of method of Peritoneal Dialysis (PD) catheter insertion. (1B)

Guideline 4.2 - PD Access: Implantation Technique

We recommend that each $\mathrm{PD}$ unit should have the ability to manipulate or re-implant PD catheters when necessary. (1B)

Guideline 4.3 - PD Access: Implantation Technique

We recommend that urgent removal of $\mathrm{PD}$ catheters should be available where necessary. (1A)
Guideline 4.4 - PD Access: Implantation Technique

We recommend that timely surgical support should be available for the review of PD patients. (1A)

\section{Peritoneal Dialysis Access (PD Access) \\ (Guidelines 5.1-5.4)}

\section{Guideline 5.1 - PD Access: Facilities}

We recommend that a dedicated area should be used for catheter insertion with appropriate staffing, suction, oxygen and patient monitoring facilities. (1A)

\section{Guideline 5.3 - PD Access: Facilities}

We suggest that no particular catheter type is proven to be better than another. $(2 \mathrm{C})$

\section{Guideline 5.3 - PD Access: Facilities}

We suggest that a catheter of a suitable size should be used. (2C)

\section{Guideline 5.4 - PD Access: Facilities}

We suggest that PD catheters should be inserted as day case procedures as long as this does not compromise the quality of care. (2C)

\section{Peritoneal Dialysis Access (PD Access) \\ (Guidelines 6.1-6.2)}

Guideline 6.1 - PD Access: Training

We recommend that PD catheter insertion training should be available to all trainees with an interest. (1C)

\section{Guideline 6.2 - PD Access: Training}

We recommend that $\mathrm{PD}$ catheter insertion should not be delegated to inexperienced unsupervised operators. (1A)

\section{Peritoneal Dialysis Access (PD Access) \\ (Guideline 7.1)}

\section{Guideline 7.1 - PD Access: Audit}

We recommend that there should be regular audit at not less than 12 monthly intervals of the outcome of catheter insertion as part of multi-disciplinary meetings of the PD team and the access operators. (1B) 
Summary of Clinical Practice Guidelines for Post-operative Care of the Kidney Transplant Recipient

1. Kidney Transplant Recipient (KTR): Organisation of outpatient follow-up (Guidelines 1.1-1.4)

\section{Guideline 1.1 - KTR: Clinic infrastructure}

We suggest that the following infrastructure should be in place for KTR follow up. (2D)

- A consultant-level health care professional should be available for every transplant clinic.

- KTRs should be reviewed in a dedicated outpatient area.

- The results of blood tests (including drug levels if possible) should be available within 24 hours.

- A formal mechanism should exist for results review by healthcare professionals within 24 hours of a clinic appointment.

- There should be access to a multidisciplinary renal team including pharmacist, dietician, social worker and psychologist.

- Patient care should be planned along principles set out in the National Service Framework.

\section{Guideline 1.2 - KTR: Clinic frequency}

We suggest that uncomplicated patients, as a general rule, may be reviewed progressively less frequently in clinic. $(2 \mathrm{C})$

- 2-3 times weekly for the first month after transplantation.

- 1-2 times weekly for months 2-3.

- Every 1-2 weeks for months 4-6.

- Every 4-6 weeks for months 6-12.

- 3-6 monthly thereafter.

\section{Guideline 1.3 - KTR: Patient access}

We suggest that all patients should have ready access to support services and results. (2C)

- All patients should have on-line access to their results via the 'Renal Patient View' service if they wish.

- All patients should have open access to the renal transplant outpatient service and have an established point of contact for enquiries.

- Patient information should be available in both written and electronic formats.
Guideline 1.4 - KTR: Chronic transplant care review

We suggest that a detailed review should be performed annually post-operatively. (2C)

- A process should exist for patient review on an annual basis in a different format of clinic according to the 'Care plan model'.

- This should be a patient-centred clinic, facilitated by a healthcare professional.

- It should address concerns in medical, social, psychological and sexual domains.

- Open access to a renal dietician, social worker, specialist renal pharmacist or psychologist should be readily available from this clinic.

- This process should proceed in parallel with formal medical review.

\section{Kidney Transplant Recipient (KTR): Non-} adherence (Guideline 2.1)

\section{Guideline 2.1 - KTR: Recognising non-adherence}

We suggest that it is important to prevent and detect non-adherence in kidney transplant recipients. (2C)

- Factors associated with non-adherence should be identified.

- An established interventional pathway should be in place for those at high risk of or with proven nonadherence.

- Pathways should be in place for paediatric KTRs in transition and for adolescent KTRs.

3. Kidney Transplant Recipient (KTR):

Immunosuppressive treatment (Guidelines 3.1-

3.15)

Guideline 3.1 - KTR: Induction immunosuppression

We recommend induction therapy should take into account the following:

- Immunosuppressive drugs should be started before or at the time of renal transplantation. (1B)

- Induction therapy with biological agents should be administered to all KTRs. (1B) In patients at low immunological risk this will generally involve an interleukin-2 receptor antagonist (IL2-RA). Recipients at higher immunological risk may be considered for T-cell (lymphocyte) Depleting Antibodies (TDAs; e.g. anti-lymphocyte preparations [ALG, ATG], alemtuzumab or OKT3). 
- Induction therapy with TDAs may also be useful for lower immunological risk patients with the intention of either steroid or CNI avoidance. (1C)

Guideline 3.2 - KTR: Induction immunosuppression

We suggest that a CNI should be started at the time of transplantation and not delayed until the graft is functioning. (2C)

\section{Guideline 3.3 - KTR: Maintenance immunosuppression}

We recommend that maintenance immunosuppression should normally consist of a calcineurin inhibitor (CNI) and an anti-proliferative agent, with or without corticosteroids in low and medium immunological risk KTRs. (1B)

\section{Guideline 3.4 - KTR: Maintenance immunosuppression}

We suggest that low dose tacrolimus (trough target $3-7 \mathrm{ng} / \mathrm{ml}$ ) is recommended as the CNI of choice in patients also taking steroids who are low and medium immunological risk and are not at high risk of developing NODAT. (2C)

\section{Guideline 3.5 - KTR: Maintenance immunosuppression}

We suggest that MPA-based drugs should be the firstline antiproliferative agent, in preference to azathioprine. (2B)

\section{Guideline 3.6 - KTR: Maintenance immunosuppression}

We suggest that mycophenolate mofetil (Cellcept ${ }^{\circledR}$ ) and enteric coated mycophenolate sodium (Myfortic ${ }^{\mathbb{R}}$ ) provide equivalent maintenance immunosuppression. (2B)

\section{Guideline 3.7 - KTR: Maintenance immunosuppression}

We suggest that vigilant steroid avoidance or steroid withdrawal can generally be used during the first week after transplantation in low immunological risk kidney transplant recipients. (2B)

\section{Guideline 3.8 - KTR: Maintenance}

\section{immunosuppression}

We suggest aiming for minimum target levels for CNIs in uncomplicated renal transplantation after 3 months. $(2 \mathrm{C})$

\section{Guideline 3.9 - KTR: Maintenance}

\section{immunosuppression}

We suggest that CNIs should be continued rather than withdrawn. (2B)

\section{Guideline 3.10 - KTR: Maintenance immunosuppression}

We suggest if steroids are not withdrawn within the first month then they should be maintained at low dose (Prednisolone $-5 \mathrm{mg}$ per day or less). (2C)

\section{Guideline 3.11 - KTR: Monitoring of immunosuppression}

We suggest that long-term monitoring of immunosuppression levels is required as follows:

- Tacrolimus and ciclosporin levels should be monitored. The initial frequency should be three times a week. Levels should also be checked when any medication with possible interactions is prescribed, the dosage is changed, the formulation is changed or when there is unexplained graft dysfunction. (2C)

- Tacrolimus should be monitored by the $\mathrm{C}_{0}$ trough level, while ciclosporin can be monitored by either $\mathrm{C}_{0}$ or $\mathrm{C}_{2}$ level. $(2 \mathrm{C})$

- Tacrolimus and ciclosporin levels should be available within 24 hours of taking blood samples in the first three months after transplantation. (2D)

- The utility of monitoring MMF $\mathrm{C}_{0}$ levels is uncertain. (2D)

- Sirolimus should be monitored by the $\mathrm{C}_{0}$ trough level. (2C)

\section{Guideline 3.12 - KTR: Prescribing and the use of} generic agents

We suggest that generic compounds should not be used unless they have been shown to be bioequivalent to branded products and have been approved by the European Agency for the Evaluation of Medicinal Products (EMEA). (2D)

\section{Guideline 3.13 - KTR: Prescribing and the use of generic agents}

We suggest that KTRs should be made aware of the existence of generics and the dangers of indiscriminate usage. (2D)

\section{Guideline 3.14 - KTR: Prescribing and the use of generic agents}

We suggest that drugs should be prescribed by brand name where unproven generic substitutes are available. (2D) 
Guideline 3.15 - KTR: Prescribing and the use of generic agents

We suggest that KTRs should be followed closely after switching to a generic preparation until a new steady state is established. (2D)

4. Kidney Transplant Recipient (KTR): Acute rejection (Guidelines 4.1-4.10)

Guideline 4.1 - KTR: Diagnosis of acute rejection

We recommend that a transplant renal biopsy should be carried out before treating an acute rejection episode unless this will substantially delay treatment or pose a significant risk to the patient. (1C)

\section{Guideline 4.2 - KTR: Diagnosis of acute rejection}

We suggest that two cores of renal tissue should be obtained if possible since this will increase the sensitivity of the investigation. (2C)

Guideline 4.3 - KTR: Diagnosis of acute rejection

We suggest that routine C4d and SV40 staining should be performed upon transplant biopsies. (2C)

\section{Guideline 4.4 - KTR: Treatment of acute rejection}

We suggest that borderline acute cellular rejection should be treated. (2D)

\section{Guideline 4.5 - KTR: Treatment of acute rejection}

We recommend that high dose intravenous corticosteroids should be the first line treatment for acute cellular rejection. (1D)

\section{Guideline 4.6 - KTR: Treatment of acute rejection}

We suggest that maintenance steroids should be added or restarted in steroid-free patients undergoing acute rejection of any type. (2D)

\section{Guideline 4.7 - KTR: Treatment of acute rejection}

We suggest that lymphocyte depleting agents may be considered for refractory acute cellular rejection or aggressive vascular cellular rejection (i.e. BANFF category 4 Type II and III). (2C)

\section{Guideline 4.8 - KTR: Treatment of acute rejection}

We suggest that antibody mediated rejection (AMR) should be treated with one or more of the following modalities: steroids; plasma exchange; intravenous immunoglobulin; anti-CD20 antibody; or lymphocytedepleting antibody. (2C)

Summary of the 5th Edition of the Renal Association Clinical Practice Guidelines
Guideline 4.9 - KTR: Treatment of acute rejection

We suggest after an episode of rejection (unless associated with low CNI levels) that azathioprine should be switched to MPA-based immunosuppression, MPA should be started or the existing dose of MPA maximised. (2D)

Guideline 4.10 - KTR: Treatment of acute rejection

We suggest that a serum sample should be sent at the time of renal biopsy (for graft dysfunction) to look for HLA-specific antibodies. (2C)

\section{Kidney Transplant Recipient (KTR): Chronic Allograft Injury (Guidelines 5.1-5.7)}

\section{Guideline 5.1 - KTR: Diagnosis of Chronic Allograft} Injury (CAI)

We recommend that early identification of graft injury is desirable to maximise the potential to intervene. A proactive and systematic approach should employed to identify graft dysfunction. (1C)

\section{Guideline 5.2 - KTR: Detection of Chronic Allograft} Injury (CAI)

We suggest that renal function should be monitored at each clinic visit by assessment of serum creatinine and qualititative evaluation of urine protein excretion by dipstick supplemented by spot PCR or ACR if positive. (2C)

\section{Guideline 5.3 - KTR: Diagnosis of Chronic Allograft} Injury (CAI)

We suggest that renal biopsy is the optimal investigation for parenchymal causes of graft dysfunction. (2C)

\section{Guideline 5.4 - KTR: Diagnosis of Chronic Allograft} Injury (CAI)

We suggest that renal biopsies in patients with chronically deteriorating function should routinely be stained for C4d and SV40. (2C)

\section{Guideline 5.5 - KTR: Diagnosis of Chronic Allograft} Injury (CAI)

We suggest that a serum sample should be sent at the time of renal biopsy (for graft dysfunction) to look for HLA-specific antibodies. (2C)

\section{Guideline 5.6 - KTR: Treatment of chronic allograft} injury

We suggest that chronic allograft injury should be treated:

Nephron Clin Pract 2011;118(suppl 1):c27-c70 
- By withdrawal of calcineurin inhibitors (CNIs) if there is either histological evidence of CNI toxicity or non-specific interstitial fibrosis and tubular atrophy. (2C)

- By intensification of immunosuppression if there is evidence of ongoing immune injury (cellular rejection and/or humoral rejection). (2C)

- In a similar fashion to other patients with CKD following similar preventative strategies and with timely referral to low clearance services. (2D)

\section{Guideline 5.7 - KTR: Renal biopsy in chronic allograft injury}

We suggest that a renal transplant biopsy is indicated:

- If there is a persistent unexplained elevation of creatinine or failure to return to baseline after an episode of BPAR. (1C)

- Every 7-10 days during DGF. (2C)

- If expected renal function is not achieved within 48 weeks. (2D)

- If sustained new onset proteinuria develops (PCR $>50$ or ACR $>35)$. (2C)

\section{Kidney Transplant Recipient (KTR): \\ Cardiovascular disease (Guidelines 6.1-6.6)}

\section{Guideline 6.1 - KTR: Hypertension}

We suggest that the management of hypertension take into account that:

- Blood pressure should be recorded at each clinic visit. (1C)

- Clinic blood pressure should be less than 130/ $80 \mathrm{mmHg}$ in clinic $(125 / 75 \mathrm{mmHg}$ if PCR $>50$ or ACR >35). (2C)

- Home blood pressure recordings and 24-hour ambulatory recordings may be helpful in some instances but lower targets should be set. (2D)

- There is no evidence that any antihypertensive agent is better than any other and effort should be focused on achieving absolute levels rather than the use of individual agents. (2D)

- Inhibitors of the renin-angiotensin system may be more effective in the minimisation of proteinuria but they should be used with caution in the first 3 months post transplant. (2C)

- Resistant hypertension may be due to transplant renal artery stenosis and should be investigated according to local practice. (2D)

\section{Guideline 6.2 - KTR: Dyslipidaemia}

We suggest that the management of dyslipidaemia take into account that:

- Fasting lipid levels should be measured on an annual basis in all renal transplant recipients. (2C)

- Treatment targets should be the same as in the general population. (2C)

- KTRs at increased primary or secondary CV risk receive statin therapy to reduce the risk of coronary artery disease. (2C)

- The choice and dose of statin should take into account concurrent immunosuppression. (2D)

\section{Guideline 6.3 - KTR: Diabetes mellitus}

We suggest that the detection and treatment of diabetes should consider:

- Screening for the development of post transplant diabetes by dipstick urinalysis and measurement of blood sugar level at each clinic visit. (2C)

- Post transplant immunosuppression should take into account risk factors for the development of diabetes. (2C)

- Post-transplant diabetes should be managed in collaboration with specialists in diabetic medicine. (2D)

- All units should have a protocol for the management of post-transplant diabetes. (2C)

\section{Guideline 6.4 - KTR: Ischaemic heart disease}

We suggest that KTRs receive standard treatment for ischaemic heart disease, including thrombolysis, revascularisation, and secondary prevention. (2C)

\section{Guideline 6.5 - KTR: Smoking cessation}

We recommend that smoking should be discouraged in transplant recipients (see guideline 6.4). (1A)

\section{Guideline 6.6 - KTR: Lifestyle measures}

We suggest that advice on healthy lifestyle forms a routine part of post-transplant care:

- Maintenance of a healthy diet should be encouraged. (2C)

- An active lifestyle should be encouraged. (2D)

- An ideal weight should be targeted (BMI $\leqslant 25)$. (2C)

- Weight management services should be available. $(2 \mathrm{C})$ 
- Alcohol consumption should be within national guidelines. (2D)

- Recreational drug use should be avoided. (2D)

- The use of over-the-counter medications (without discussion with clinical staff ) and non-proprietary medications (e.g. herbal medicines) should be discouraged. (2D)

\section{Kidney Transplant Recipient (KTR): Neoplasia}

(Guidelines 7.1-7.9)

\section{Guideline 7.1 - KTR: Screening for cancer}

We suggest that the organisation of screening for neoplasia in KTRs take into account:

- Screening should be similar to the general population for cervical, breast, colon and prostate cancer. (2C)

- Screening is not recommended for renal cell carcinoma. (2C)

- Breast and testicular self-examination should be encouraged. (2D)

- An annual examination of skin by a healthcare professional. (2C)

- Patients with cirrhosis should undergo an annual hepatic ultrasound and determination of serum alpha feto-protein. (2C)

\section{Guideline 7.2 - KTR: Non-Melanoma Skin Cancer} (NMSC)

We recommend that KTRs should be educated about the adverse effects of solar exposure. (1C)

\section{Guideline 7.3 - KTR: Non-Melanoma Skin Cancer (NMSC)}

We suggest that an individualised assessment of hazard should be made according to risk factors. (2C)

\section{Guideline 7.4 - KTR: Non-Melanoma Skin Cancer (NMSC)}

We recommend that patients should be encouraged to cover their skin in direct sunlight and to use total sunblock (Sun Protection Factor $\geqslant 50$ ). (1D)

\section{Guideline 7.5 - KTR: Non-Melanoma Skin Cancer (NMSC)}

We suggest that self examination should be encouraged and should be supplemented by annual review by a trained healthcare professional. (2C)
Guideline 7.6 - KTR: Non-Melanoma Skin Cancer (NMSC)

We suggest that acitretin should be prescribed to those with previous NMSC if there are no contraindications. (2B)

Guideline 7.7 - KTR: Immunosuppression in cancers We suggest that the overall level of immunosuppression should be reduced if neoplasia develops. (2C)

Guideline 7.8 - KTR: Immunosuppression in cancers We suggest that $\mathrm{m}$-TORis are considered as alternative immunosuppressive agents in KTRs who develop de novo maligancy. (2C)

\section{Guideline 7.9 - KTR: Immunosuppression in} Kaposi's sarcoma

We suggest that m-TORis have specific anti-tumour effects in Kaposi's sarcoma and switching to this medication should be considered. (2C)

\section{Kidney Transplant Recipient (KTR): Infection Complications (Guidelines 8.1-8.7)}

\section{Guideline 8.1 - KTR: Vaccination}

\section{Guideline 8.1.1 - KTR: Vaccination}

We recommend that KTRs:

- Should be vaccinated with inactivated viruses as per the normal population except for HBV. (1D)

- Should receive annual influenza vaccination unless contraindicated. (1C)

\section{Guideline 8.1.2 - KTR: Vaccination}

We suggest that KTRs:

- Should have HBsAb levels rechecked annually and revaccination carried out if antibody titres fall below $10 \mathrm{mIU} / \mathrm{ml}$. (2D)

- Should not receive live attenuated vaccines. (2C)

- Should receive pneumococcal vaccine and one booster every five years. (2D)

Guideline 8.2 - KTR: Cytomegalovirus disease

Guideline 8.2.1 - KTR: Prophylaxis and treatment of CMV disease

We recommend: 
- Prophylaxis should be continued for 3-6 months, until immunosuppression has been reduced to long-term maintenance level; 6 months has proven benefit in sero-negative recipients of kidneys from CMV positive donors. (1B)

- Treatment should be administered for 6 weeks after treatment with a TDA. (1C)

\section{Guideline 8.2.2 - KTR: Prophylaxis and treatment of CMV disease}

We suggest:

- All transplant units should have the ability to measure CMV serological status and the detection and quantification of viral load. (2D)

- Donor and recipient CMV sero-positivity should be recorded at the time of transplantation. (2D)

- A written protocolised strategy based either on prophylaxis, or pre-emptive therapy, or both should be implemented. (2D)

- For the treatment of mild and moderate CMV disease, oral valganciclovir and intravenous ganciclovir are of equivalent efficacy. (2C)

- Treatment of life-threatening CMV disease should be initiated with intravenous ganciclovir. (2D)

- Treatment duration should be determined by monitoring viral load. (2C)

\section{Guideline 8.3 - KTR: Epstein Barr Virus infection}

\section{Guideline 8.3.1 - KTR: EBV infection}

We recommend that immunosuppression should be reduced or stopped following the development of PTLD. (1C)

\section{Guideline 8.3.2 - KTR: EBV infection}

We suggest:

- Both donor and recipient should have their EBV serology recorded at the time of transplantation. (2D)

- All high risk $\left(\mathrm{D}^{+} / \mathrm{R}^{-}\right)$patients (including adults) should have EBV viral load measured immediately after transplantation, monthly for six months, and three monthly to one year. (2C)

- EBV viral load should be monitored after the treatment of rejection. (2C)

- Total immunosuppression should be reduced when EBV titres rise significantly. (2C)
Guideline 8.4-KTR: Varicella Zoster Virus infection

\section{Guideline 8.4.1 - KTR: VZV infection}

We recommend:

- Primary infection (chickenpox) should be treated with intravenous aciclovir or oral valaciclovir until the lesions scab over. (1C)

- Uncomplicated shingles should be treated with oral acyclovir or valaciclovir until the lesions scab over. (1D)

- Disseminated ( $>2$ dermatomes), ocular or invasive shingles should be treated with intravenous aciclovir until the lesions scab over, together with a reduction in immunosuppression. (1B)

- Varicella-susceptible KTRs (i.e. VZV IgG -ve) with primary exposure to VZV should receive intravenous immunoglobulins, ideally within 96 hours, but up to a maximum of 10 days following exposure. If unavailable or after 10 days, oral aciclovir should be administered for seven days, starting one week after exposure. (1D)

\section{Guideline 8.4.2 - KTR: VZV infection}

We suggest:

- Patients on the waiting list who are VZV IgG negative should be vaccinated prior to transplantation. (2D)

- Immunosuppression should be reduced during primary infection. (2D)

\section{Guideline 8.5 - KTR: Herpes Simplex Virus infection}

\section{Guideline 8.5.1 - KTR: HSV infection}

We recommend:

- Superficial HSV infection should be treated with appropriate oral agents until the lesions have resolved. (1D)

- Systemic HSV infections should be treated with intravenous aciclovir and a reduction in immunosuppression until a response occurs and oral medication continued for at least 14 days. (1C)

\section{Guideline 8.5.2 - KTR: HSV infection}

We suggest that KTRs suffering frequent recurrent HSV infection should consider oral prophylaxis. (2D) 


\section{Guideline 8.6 - KTR: BK nephropathy}

\section{Guideline 8.6.1 - KTR: BK nephropathy}

We recommend that confirmed BK nephropathy should be treated by reduction in immunosuppression. (1D)

\section{Guideline 8.6.2 - KTR: BK nephropathy}

We suggest:

- KTRs should be screened for BKV viral load by performing urine microscopy for decoy cells or by PCR on urine or serum. (2C)

- Screening should be monthly for the first six months, then every three months until the end of the first year. (2D)

- Screening should also be carried out when renal function deteriorates in an unexplained fashion or when immunosuppression is intensified. (2D)

- Suspected BK nephropathy should be confirmed by renal biopsy which should be stained for SV40. Two cores containing medullary tissue should ideally be examined. (2D)

- Immunosuppression should be reduced when the serum BKV load exceeds $10^{4}$ copies $/ \mathrm{ml}$. (2C)

- There is no established specific treatment for BK nephropathy. (2D)

- Re-transplantation can safely be considered in patients who have BK nephropathy diagnosed in an earlier graft. (2C)

\section{Guideline 8.7 - KTR: Post-transplant infection prophylaxis}

We suggest:

- All patients should receive 3-6 months of treatment with co-trimoxazole $480 \mathrm{mg}$ daily. (1B)

- Oral antifungal prophylaxis should be administered for three months after transplantation. (2C)

- In selected patients, prophylaxis against mycobacterium tuberculosis with daily isoniazid (supplemented with pyridoxine) should be instituted for six months after transplantation. (2C)

\section{Kidney Transplant Recipient (KTR): Bone and joint disease (Guidelines 9.1-9.4)}

\section{Guideline 9.1 - KTR: Osteoporosis}

We suggest:
- KTRs suffering from osteoporosis or at high potential risk should be considered for steroid-avoiding immunosuppression. (2D)

- KTRs on longterm steroids or at high risk for osteoporosis should undergo DEXA scanning if eGFR $>30 \mathrm{ml} / \mathrm{min} / 1.73 \mathrm{~m}^{2}$. (2D)

- Treatment should be according the RCP guidelines for steroid-induced osteoporosis. (2D)

Guideline 9.2 - KTR: Tertiary hyperparathyroidism We suggest:

- Severe hyperparathyroidism should be treated prior to transplantation. (2D)

- Cinacalcet can be used in KTRs. (2C)

- Treatment should be the same as for other patients with CKD. (2D)

\section{Guideline 9.3 - KTR: Gout}

\section{Guideline 9.3.1 - KTR: Treatment of gout}

We recommend that allopurinol should not be administered with azathioprine. (1B)

\section{Guideline 9.3.2 - KTR: Treatment of gout}

We suggest:

- Hyperuricaemia should be treated when associated with gout, tophi or uric acid stones. (2D)

- Non steroidal anti-inflammatory drugs (NSAIDs) should be avoided in KTRs. (2D)

- Episodes of gout may be treated with brief courses of oral prednisolone. (2D)

- Colchicine is an effective treatment for gout in KTRs. (2D)

\section{Guideline 9.4 - KTR: Calcineurin inhibitor bone pain}

We suggest:

- Reducing or withdrawing CNIs should be considered in KTRs with intractable bone pain. (2D)

- Dihydropyridine calcium antagonists also may be beneficial. (2D)

10. Kidney Transplant Recipient (KTR): Haematological complications (Guidelines 10.110.3)

\section{Guideline 10.1 - KTR: Anaemia}

We suggest that anaemia should be managed in the same way as other patients with CKD. (2D) 
Guideline 10.2 - KTR: - Polycythaemia

We recommend that initial treatment should be with angiotensin converting enzyme inhibitors (ACEIs) or with angiotensin receptor blockers (ARBs). (1C)

\section{Guideline 10.3 - KTR: - Polycythaemia}

We suggest:

- Haemoglobin levels should be monitored at every clinic visit. (2D)

- Treatment should be initiated if the haematocrit or packed cell volume exceeds 52\% in men and $49 \%$ in women. (2D)

- Aminophylline and venesection may be used in refractory cases. (2D)

\section{Kidney Transplant Recipient (KTR):}

Reproductive issues (Guidelines 11.1-11.5)

\section{Guideline 11.1 - KTR: Conception and contraception (female)}

We recommend that MPA-containing immunosuppressant drugs should be stopped prior to conception and replaced appropriately. (1A)

\section{Guideline 11.2 - KTR: Conception and contraception (female)}

We suggest:

- KTRs should wait for one year after transplant and have stable function before attempting conception. (2C)

- Counselling regarding fertility and reproduction should be offered to female KTRs and their partners either prior to transplantation or soon afterwards. (2D)

- m-TORi should be stopped prior to conception and replaced as appropriate. (2D)

- Pregnancy should be managed jointly with an Obstetrics department. (2D)
- The risks and benefits of breastfeeding should be discussed. (2D)

- Contraception advice should be similar to the general population. (2D)

\section{Guideline 11.3 - KTR: Conception (male)}

We recommend that KTRs should be advised that mTORi reduce the male sperm count and counselled accordingly. (1C)

\section{Guideline 11.4 - KTR: Conception (male)}

We suggest:

- All immunosuppressive drugs other than m-TORi can be used in male KTRs. (2D)

- Men on m-TORi who wish to conceive should discontinue these agents prior to conception and replace them as appropriate. (2D)

- Men who wish to maintain fertility should avoid m-TORi or bank sperm prior to starting these drugs. m-TORi reduce the male sperm count and KTRs should be counselled accordingly. (2D)

- Men should be counselled about the possible risks of impotence following transplantation surgery that involves the internal iliac artery. (2D)

\section{Guideline 11.5 - KTR: Sexual dysfunction}

We suggest:

- Specific enquiry should be made regarding sexual dysfunction, preferably at an annual review clinic. (2D)

- Care pathways for dealing with sexual dysfunction should be established. (2D)

- Close liaison with the local andrology service is recommended. (2D)

- Sildenafil is safe and effective in male KTRs not taking nitrates. (2D) 


\section{Summary of Clinical Practice Guideline on Acute Kidney Injury}

\section{Acute Kidney Injury (AKI) (Guidelines AKI} 1.1-1.3)

\section{Guideline 1.1 - AKI: Definition, Epidemiology and} Outcomes

We recommend that the international Kidney Disease: Improving Global Outcomes (KDIGO) definition of acute kidney injury (AKI) should be adopted. (Not Graded)

Acute kidney injury is defined when one of the following criteria is met

- Serum creatinine rises by $\geqslant 26 \mathrm{~mol} / \mathrm{L}$ within 48 hours or

- Serum creatinine rises $\geqslant 1.5$ fold from the reference value, which is known or presumed to have occurred within one week or

- urine output is $<0.5 \mathrm{ml} / \mathrm{kg} / \mathrm{hr}$ for $>6$ consecutive hours

The reference serum creatinine should be the lowest creatinine value recorded within 3 months of the event

If a reference serum creatinine value is not available within 3 months and AKI is suspected

- repeat serum creatinine within 24 hours

- a reference serum creatinine value can be estimated from the nadir serum creatinine value if patient recovers from AKI

\section{Guideline 1.2 - AKI: Definition, Epidemiology and Outcomes}

We recommend that the international Kidney Disease: Improving Global Outcomes (KDIGO) staging classification $^{*}$ (see the table below) of acute kidney injury (AKI) should be adopted. (Not Graded)
Guideline 1.3 - AKI: Definition, Epidemiology and Outcomes

We recommend that serum creatinine and urine output remain the best biomarkers for AKI. Serum creatinine should be measured using the enzymatic technique. (1B)

\section{Acute Kidney Injury (AKI) (Guidelines AKI}

2.1-2.2)

Guideline 2.1 - AKI: Clinical Assessment; History, Examination

We recommend that all patients presenting with AKI should have a comprehensive history and examination performed to help determine the aetiology of the AKI. (1A)

\section{Guideline 2.2 - AKI: Clinical Assessment; Investigations}

We recommend that all patients presenting with AKI should have appropriate baseline investigations performed which should include a urinalysis and a renal tract ultrasound within 24 hours (if renal tract obstruction is suspected). (1A)

\section{Acute Kidney Injury (AKI) (Guidelines AKI 3.1-3.4)}

\section{Guideline 3.1 - AKI: Prevention; Risk Assessment}

We recommend that patients at risk of AKI should be identified and appropriate preventative measures should be instituted as early as possible. (1B)

\section{Guideline 3.2 - AKI: Prevention; Fluid Therapy}

We recommend that prescription of appropriate intravenous fluid should be carefully considered following assessment of the patient's volume status. Thereafter the patient's clinical response should be monitored closely. (1B)

\begin{tabular}{lll}
\hline Stage & Serum creatinine $(\mathrm{SCr})$ criteria & Urine output criteria \\
\hline 1 & $\begin{array}{l}\text { increase } \geqslant 26 \mu \mathrm{mol} / \mathrm{L} \text { within } 48 \mathrm{hrs} \text { or } \\
\text { increase } \geqslant 1.5 \text { to } 1.9 \mathrm{X} \text { reference SCr }\end{array}$ & $<0.5 \mathrm{~mL} / \mathrm{kg} / \mathrm{hr}$ for $>6$ consecutive hrs \\
2 & increase $\geqslant 2$ to $2.9 \mathrm{X}$ reference SCr & $<0.5 \mathrm{~mL} / \mathrm{kg} / \mathrm{hr}$ for $>12 \mathrm{hrs}$ \\
3 & $\begin{array}{l}\text { increase } \geqslant 3 \mathrm{XL} / \mathrm{kg} / \mathrm{hr} \text { for }>24 \mathrm{hrs} \text { or anuria for } 12 \mathrm{hrs} \\
\text { increase } \geqslant 354 \mu \mathrm{mol} / \mathrm{L} \text { or } \text { or } \\
\text { commenced on renal replacement therapy (RRT) irrespective of stage }\end{array}$ & \\
\hline
\end{tabular}

${ }^{*}$ Must have met initial criteria for definition of AKI 
Guideline 3.3 - AKI: Prevention; Contrast-Induced AKI (CI-AKI)

We recommend that patients identified as being at risk of contrast induced-AKI (CI-AKI) should have a careful assessment of volume status and receive pre-procedure volume expansion with $0.9 \%$ sodium chloride or isotonic sodium bicarbonate if clinically indicated. (1A)

Guideline 3.4 - AKI: Prevention; AKI secondary to Rhabdomyolysis

We recommend that patients identified as being at risk of developing AKI secondary to rhabdomyolysis should receive intravenous volume expansion with $0.9 \%$ sodium chloride and sodium bicarbonate. (1B)

\section{Acute Kidney Injury (AKI) (Guidelines AKI} 4.1-4.5)

\section{Guideline 4.1 - AKI: Management; General Management}

We recommend that general supportive measures include optimisation of haemodynamic status by appropriate fluid therapy, administration of vasopressors and/ or inotropes and treatment of any underlying sepsis. Nephrotoxic medications should be stopped. (1A)

Guideline 4.2 - AKI: Management; Pharmacological Therapy

We recommend that therapeutic drug dosing must be adapted to altered kinetics in AKI. (1B)

Guideline 4.3 - AKI: Management; Pharmacological Therapy

We recommend that there is no specific pharmacological therapy proven to effectively treat AKI secondary to hypoperfusion injury and/or sepsis. (1B)

\section{Guideline 4.4 - AKI: Management; Nutritional Support}

We recommend that patients with AKI receiving renal replacement therapy (RRT) should be referred to a dietician for individual assessment. (1D)

\section{Guideline 4.5 - AKI: Management; Nutritional support}

We recommend that patients with AKI should receive $25-35 \mathrm{kcal} / \mathrm{kg} /$ day and up to a maximum of $1.7 \mathrm{~g}$ amino acids $/ \mathrm{kg} /$ day if hypercatabolic and receiving continuous renal replacement therapy. Trace elements and water soluble vitamins should be supplemented as required. (1C)

\section{Acute Kidney Injury (AKI) (Guidelines AKI 5.1-5.7)}

Guideline 5.1 - AKI: Treatment facilities and referral to renal services

We recommend that renal services should work together with other specialties to develop guidelines for the management of AKI. These should include clear guidelines with respect to when to request a renal referral. (1A)

Guideline 5.2 - AKI: Treatment facilities and referral to renal services

We recommend that specialist renal advice should be given with consultant renal physician input. (1B)

\section{Guideline 5.3 - AKI: Treatment facilities and referral} to renal services

We recommend that transfer protocols should be developed based on local physiological early warning scores to ensure appropriate triage of in-patients with AKI arriving from other hospitals. (1C)

\section{Guideline 5.4-AKI: Treatment facilities and referral} to renal services

We recommend that physiological surveillance should be performed for all patients with AKI to identify early signs of physiological deterioration which may require escalation in the level of care. $(1 \mathrm{~A})$

\section{Guideline 5.5 - AKI: Treatment facilities and referral to renal services}

We suggest that renal physicians and intensivists should work together to provide care for patients with AKI on the intensive care unit (ICU). Nephrology trainees should be trained to care for acutely ill patients with AKI. (2C)

\section{Guideline 5.6 - AKI: Treatment facilities and referral} to renal services

We suggest that intensive care units should contact renal services to discuss patients likely to require ongoing single organ renal support prior to step-down. Advance warning of such patients will facilitate forward planning and continued follow-up. (2C)

\section{Guideline 5.7 - AKI: Treatment facilities and referral} to renal services

We recommend that AKI survivors with residual renal impairment should be managed according to local chronic kidney disease (CKD) guidelines. Discharge 
planning should include plans for CKD management, where relevant. (1A).

\section{Acute Kidney Injury (AKI) (Guideline AKI 6.1)}

\section{Guideline 6.1 - AKI: Choice of renal replacement} therapy modality

We recommend that the choice of renal replacement therapy modality should be guided by the individual patient's clinical status, medical and nursing expertise, and availability of modality. (1B)

\section{Acute Kidney Injury (AKI) (Guidelines AKI} 7.1-7.3)

\section{Guideline 7.1 AKI: Choice of dialyser / haemofilter membrane}

We recommend that synthetic or modified cellulosic membranes should be used in preference to unmodified cellulose membranes. (1B)

\section{Guideline 7.2 AKI: Choice of dialysate / replacement fluid}

We recommend that bicarbonate should be the preferred buffer for dialysate and replacement fluid in continuous renal replacement therapy (CRRT) techniques unless regional citrate anticoagulation is employed. (1C)

\section{Guideline 7.3 AKI: Microbial standards for fluids}

We recommend that microbial standards for fluids used for chronic haemodialysis (HD)/haemodiafiltration (HDF) should be also applied to extracorporeal therapy for AKI. (1A)

\section{Acute Kidney Injury (AKI) (Guidelines AKI 8.1-8.9)}

\section{Guideline 8.1 - AKI: Vascular access for RRT}

We recommend that acute access for renal replacement therapy should be veno-venous rather than arterio-venous. (1A)

\section{Guideline 8.2 - AKI: Vascular access for RRT}

We recommend that dialysis catheters should be of an adequate length to minimise the risks of access recirculation. (1C)

\section{Guideline 8.3 - AKI: Vascular access for RRT}

We suggest that the access site and catheter type should be chosen with regard to the phase of the patient's illness. (2C)
Guideline 8.4 - AKI: Vascular access for RRT

We recommend that access should be placed by experienced or appropriately supervised staff. Real-time ultrasound guidance should be used to aid placement of upper body access. (1A)

\section{Guideline 8.5 - AKI: Vascular access for RRT}

We recommend that it is advisable that real-time ultrasound guidance be used for the insertion of femoral access. (1D)

\section{Guideline 8.6 - AKI: Vascular access for RRT}

We recommend that subclavian access should be avoided in patients at risk of progressing to CKD stage 4 or 5 due to the risks of compromising future, permanent vascular access. (1D)

\section{Guideline 8.7 - AKI: Vascular access for RRT}

We suggest that non-dominant arm upper limb vasculature should be preserved as a contingency for future permanent access. (2C)

\section{Guideline 8.8 - AKI: Vascular access for RRT}

We recommend that temporary access should be changed at appropriate intervals (as per local protocol) to minimise the risk of infection. (1C)

\section{Guideline 8.9 - AKI: Vascular access for RRT}

We suggest that local policies on prevention of catheter-related infection should be optimised by reserving the catheter for extracorporeal treatment only. (2D)

\section{Acute Kidney Injury (AKI) (Guidelines AKI 9.1-9.4)}

\section{Guideline 9.1 - AKI: Anticoagulation for extracorporeal therapies}

We recommend that anticoagulation for RRT should be tailored according to patient characteristics and the modality of RRT chosen. (1C)

\section{Guideline 9.2 - AKI: Anticoagulation for extracorporeal therapies}

We recommend that regional anticoagulation with citrate reduces risk of haemorrhage compared to systemic heparinisation. The complexity of the technique means that this should be in routine use on any unit on which it is employed in order to allow sufficient levels of expertise to be maintained. (1C)

Nephron Clin Pract 2011;118(suppl 1):c27-c70 
Guideline 9.3 - AKI: Anticoagulation for

extracorporeal therapies

We suggest that prostacyclin is a suitable alternative to unfractionated heparin in those at increased risk of bleeding but may cause haemodynamic instability. (2C)

\section{Guideline 9.4 - AKI: Anticoagulation for}

\section{extracorporeal therapies}

We suggest that a no-anticoagulation, saline flush strategy can be used in patients receiving continuous and intermittent RRT who are at high risk of bleeding. However, ultrafiltration requirements are increased, effective intermittent HD time is reduced and the technique runs the risk of membrane fibre rupture. $(2 \mathrm{C})$

\section{Acute Kidney Injury (AKI) (Guidelines AKI 10.1-10.5)}

\section{Guideline 10.1 - AKI: Renal Replacement Therapy prescription}

We recommend that the delivered dose of RRT should be assessed to ensure the adequacy of the prescription. (1A)

\section{Guideline 10.2 - AKI: Renal Replacement Therapy prescription}

We recommend that the prescribed dose should be assessed at each session (for intermittent haemodialysis) and daily (for continuous RRT) to account for any measured shortfalls in delivered dose. (1A)

\section{Guideline 10.3 - AKI: Renal Replacement Therapy prescription}

We recommend that patients with AKI and multiorgan failure treated by continuous renal replacement therapy (CRRT) should receive treatment doses equivalent to post dilution ultrafiltration rates $\geqslant 25 \mathrm{ml} / \mathrm{kg} / \mathrm{hr}$. A proportionate upward adjustment to the prescribed ultrafiltration rate should be made in pre-dilutional continuous haemofiltration. (1A)

\section{Guideline 10.4 - AKI: Renal Replacement Therapy prescription}

We recommend that patients with AKI and multiorgan failure treated by intermittent haemodialysis should receive either alternate day haemodialysis with at least the minimum dose considered appropriate for end-stage renal disease (ESRD), urea reduction ratio (URR) $>65 \%$ or eKt/V $>1.2$ or daily haemodialysis. (1B)
Guideline 10.5 - AKI: Renal Replacement Therapy prescription

We suggest that renal replacement therapy dosing methods that require an assessment of patient weight should use a measured weight rather than an extrapolated weight from pre-morbid readings. (2B)

\section{Acute Kidney Injury (AKI) (Guidelines AKI 11.1-11.5)}

Guideline 11.1 - AKI: Timing of initiation of renal replacement treatment

We recommend that the decision to start RRT in patients with AKI should remain a clinical decision based on fluid, electrolyte and metabolic status of each individual patient. (1C)

\section{Guideline 11.2 - AKI: Timing of initiation of renal} replacement treatment

We recommend that RRT should be initiated once AKI is established and unavoidable but before overt complications have developed. (1B)

\section{Guideline 11.3 - AKI: Timing of initiation of renal} replacement treatment

We recommend that the threshold for initiating RRT should be lowered when AKI occurs as part of multiorgan failure. (1C)

Guideline 11.4 - AKI: Timing of initiation of renal replacement treatment

We recommend that the initiation of RRT may be deferred if the underlying clinical condition is improving and there are early signs of renal recovery. (1D)

\section{Guideline 11.5 - AKI: Timing of discontinuation of} renal replacement treatment

We recommend that an improvement in the patient's clinical condition and urine output would justify temporary discontinuation of ongoing renal support to see if AKI is recovering. (1D)

\section{Acute Kidney Injury (AKI) (Guidelines AKI 12.1)}

\section{Guideline 12.1 - AKI: Education}

We recommend that undergraduate and postgraduate medical trainees should be taught the principles of prevention, recognition and management of AKI. (1C) 